\title{
CNS Distribution of Members of the Two-Pore-Domain (KCNK) Potassium Channel Family
}

\author{
Edmund M. Talley, ${ }^{1}$ Guillermo Solórzano, ${ }^{1}$ Qiubo Lei, ${ }^{1}$ Donghee Kim, ${ }^{2}$ and Douglas A. Bayliss ${ }^{1}$ \\ ${ }^{1}$ Department of Pharmacology, University of Virginia, Charlottesville, Virginia 22908, and ${ }^{2}$ Department of Physiology and \\ Biophysics, Finch University of Health Sciences, The Chicago Medical School, North Chicago, Illinois 60064
}

\begin{abstract}
Two-pore-domain potassium $\left(\mathrm{K}^{+}\right)$channels are substrates for resting $\mathrm{K}^{+}$currents in neurons. They are major targets for endogenous modulators, as well as for clinically important compounds such as volatile anesthetics. In the current study, we report on the CNS distribution in the rat and mouse of mRNA encoding seven two-pore-domain $\mathrm{K}^{+}$channel family members: TASK-1 (KCNK3), TASK-2 (KCNK5), TASK-3 (KCNK9), TREK-1 (KCNK2), TREK-2 (KCNK10), TRAAK (KCNK4), and TWIK-1 (KCNK1). All of these genes were expressed in dorsal root ganglia, and for all of the genes except TASK-2, there was a differential distribution in the CNS. For TASK-1, highest mRNA accumulation was seen in the cerebellum and somatic motoneurons. TASK-3 was much more widely distributed, with robust expression in all brain regions, with particularly high expression in somatic motoneurons, cerebellar granule neu-
\end{abstract}

As their name suggests, "leak" potassium $\left(\mathrm{K}^{+}\right)$channels are $\mathrm{K}^{+}$-selective and show relatively little time and voltage dependence. In neurons, they are a major determinant not only of membrane potential but also membrane input resistance, which is a primary factor in the magnitude and kinetics of responses to synaptic inputs. Thus, they are a key component in shaping the characteristics of neuronal excitability.

A family of genes (designated KCNK by the Human Genome Organization) that gives rise to leak $\mathrm{K}^{+}$channels has been identified, and over the last few years, a number of the family members have been cloned and characterized (for review, see Lesage and Lazdunski, 2000; Goldstein et al., 2001; Patel and Honore, 2001). These "two-pore-domain" $\mathrm{K}^{+}$channels are named for their predicted membrane topology, which entails four transmembrane-spanning regions surrounding two pore-forming loops. They differ structurally from members of the other major potassium channel families [the voltage-gated $\left(\mathrm{K}_{\mathrm{V}}\right)$ and inwardly rectifying $\left(\mathrm{K}_{\mathrm{IR}}\right) \mathrm{K}^{+}$channels], which consist of subunits that contain only one pore domain each.

Currently, 11 mammalian two-pore-domain $\mathrm{K}^{+}$channel family members have been demonstrated to form functional channels at the plasma membrane; of these, eight are expressed at appreciable

Received May 3, 2001; revised July 3, 2001; accepted July 26, 2001.

This work was supported by National Institutes of Health Grants NS33583 (D.A.B.) and MH12091 (predoctoral fellowship to E.M.T.). We thank Drs. M. B. Harrison, R. L. Stornetta, P. G. Guyenet, and the Information Technology Services at the University of Virginia for providing imaging equipment and support. We also thank Drs. A. T. Gray and M. Lazdunski for gifts of cDNAs.

Correspondence should be addressed to Edmund M. Talley, Department of Pharmacology, University of Virginia Health System, P.O. Box 800735, Charlottesville, VA 22908-0735. E-mail: emt3m@virginia.edu.

Copyright (C) 2001 Society for Neuroscience $0270-6474 / 01 / 217491-15 \$ 15.00 / 0$ rons, the locus ceruleus, and raphe nuclei and in various nuclei of the hypothalamus. TREK-1 was highest in the striatum and in parts of the cortex (layer IV) and hippocampus (CA2 pyramidal neurons). mRNA for TRAAK also was highest in the cortex, whereas expression of TREK-2 was primarily restricted to the cerebellar granule cell layer. There was widespread distribution of TWIK-1, with highest levels in the cerebellar granule cell layer, thalamic reticular nucleus, and piriform cortex. The differential expression of each of these genes likely contributes to characteristic excitability properties in distinct populations of neurons, as well as to diversity in their susceptibility to modulation.

Key words: potassium channel; in situ hybridization; KCNK; TASK; TREK; TRAAK; TWIK

levels in the CNS (see Discussion). The physiological similarity of these channels and their rather nondescript properties are suggestive of an invariant background role in cellular function. However, these channels appear to represent important loci for modulation of neuronal output as a result of the actions of a host of endogenous and exogenous agents (for review, see Lesage and Lazdunski, 2000; Goldstein et al., 2001; Patel and Honore, 2001). Such agents include neurotransmitters, common intracellular second messengers, a variety of bioactive lipids, and a number of clinically valuable anesthetic and neuroprotective compounds. Moreover, these channels are subject to regulation by changes in biologically important physicochemical parameters, including variations in temperature, intracellular/extracellular $\mathrm{pH}$, oxygen tension, and changes in osmolarity and/or membrane stretch (for review, see Lesage and Lazdunski, 2000; Goldstein et al., 2001; Patel and Honore, 2001). In addition, there is evidence that one of these channels (TASK-1) is a locus of modulation, in the form of compensatory expression, in neurons of mice with genetic deletions of specific GABA receptor subunits (Brickley et al., 2001).

The fact that these channels are subject to alteration from so many different sources makes them interesting from the standpoint of CNS function. Furthermore, differential modulation is a source of heterogeneity among two-pore-domain $\mathrm{K}^{+}$channel family members and is likely to contribute to diversity in pharmacological and physiological properties of CNS neurons. For each of these channels, there are data regarding CNS expression; however, in most cases, there is little detail regarding precise cellular and regional distribution. To further understand the nature of their actions in the CNS, we have localized the expression of seven of the two-pore-domain $\mathrm{K}^{+}$channel family members by in situ hybridization. 


\section{MATERIALS AND METHODS}

\section{Tissue preparation}

Adult male rats (200-350 gm) from two strains, Wistar (Harlan, Indianapolis, IN) and Sprague Dawley (Hilltop Lab Animals, Scottdale, PA), and C57BL/6J mice (The Jackson Laboratory, Bar Harbor, ME) were anesthetized with ketamine-xylazine and decapitated. Brains, spinal cords, and ganglia were removed and frozen on dry ice. Sections $(10 \mu \mathrm{m})$ were thaw mounted onto charged slides (Superfrost Plus; Fisher Scientific, Houston, TX) and stored at $-80^{\circ} \mathrm{C}$ for in situ hybridization. Hybridization was performed as described previously (Talley et al., 2000). Sections were fixed very briefly ( $5 \mathrm{~min}$ ) in $4 \%$ paraformaldehyde, rinsed repeatedly in PBS, treated successively with glycine ( $0.2 \%$ in PBS) and acetic anhydride $(0.25 \%$ in $0.1 \mathrm{M}$ triethanolamine, $0.9 \%$ saline, $\mathrm{pH} 8)$, and dehydrated in a graded series of ethanols and chloroform. Hybridization was performed overnight at $60^{\circ} \mathrm{C}$ in a buffer of $50 \%$ formamide, $4 \times$ SSC $(1 \times$ SSC: $150 \mathrm{~mm} \mathrm{NaCl}$ and $15 \mathrm{~mm}$ sodium citrate, $\mathrm{pH} 7), 1 \times$ Denhardt's solution $(0.02 \%$ each of Ficoll, polyvinylpyrrolidone, and bovine serum albumin), $10 \%$ dextran sulfate, $100 \mathrm{~mm}$ DTT, $250 \mu \mathrm{g} / \mathrm{ml}$ yeast tRNA, and $0.5 \mathrm{mg} / \mathrm{ml}$ salmon testes DNA. After hybridization, slides were washed through two changes of $4 \times$ SSC, treated with RNase A $(50 \mu \mathrm{g} / \mathrm{ml})$, washed again through two changes each of $2 \times$ SSC and $0.5 \times$ SSC, and finally subjected to a high-stringency wash of $0.1 \times$ SSC. Each of these washes lasted $20-30 \mathrm{~min}$. They were performed at $37^{\circ} \mathrm{C}$ and included $10 \mathrm{~mm}$ sodium thiosulphate, with the exception of the high-stringency wash, which was performed at $55^{\circ} \mathrm{C}$ (no sodium thiosulphate). In control experiments, the high-stringency wash was performed at different temperatures and included $25 \%$ formamide (see below).

\section{Radioactive probes}

cRNA probes were transcribed using SP6 or T7 RNA polymerase, as indicated, in the presence of $\left[\alpha-{ }^{33} \mathrm{P}\right] \mathrm{UTP}$. The templates for transcription were as follows.

TASK-1. The coding region of rat TASK-1 [obtained from A. T. Gray (University of California, San Francisco, CA); GenBank accession number AF031384; Leonoudakis et al., 1998] was ligated into pcDNA3 (Invitrogen, San Diego, CA) using Eco RI and ApaI, and linearized with HindIII for transcription using SP6 RNA polymerase. A corresponding sense probe was produced by linearizing with EcoRI and transcribing with T7.

$T A S K-2$. A mouse clone corresponding to the coding region of TASK-2 was obtained by PCR using an expressed sequence tag clone as a template (GenBank accession number AW321680; obtained from Incyte Genomics, St. Louis, MO) and ligated into pcDNA3 using BamHI and $X$ XhoI. The resulting construct was cut with HindIII and transcribed with SP6. A corresponding sense probe was generated by linearizing with $X h o I$ and transcribing with T7.

TASK-3. The coding region of rat TASK-3 was produced by PCR using cloned TASK-3 cDNA as a template (GenBank accession number AF192366; Kim et al., 2000) and ligated into pcDNA3 using BamHI and EcoRI. The resulting construct was cut with HindIII and transcribed with SP6. A corresponding sense probe was generated by linearizing with EcoRV and transcribing with T7.

TREK-1. cDNA for TREK-1 was generated by PCR from reversetranscribed mouse cerebellar RNA using primers from the sequence originally identified as the mouse TREK-1 coding region (GenBank accession number U73488.1; nucleotides 484-1596; Fink et al., 1996). This PCR product was ligated into pcRII (Invitrogen); the resulting construct lacks the final 90 nucleotides from the $\mathrm{C}$-terminal end of the revised TREK-1 coding region (GenBank accession number U73488.2). The construct was linearized using XhoI and transcribed using SP6.

TREK-2. A HindIII-Eco RI fragment of rat TREK-2 (GenBank accession number AF196965; Bang et al., 2000) was ligated into pcDNA3 (in reverse orientation); the resulting construct was linearized with EcoRI and transcribed with T7. A corresponding sense probe was generated by linearizing with HindIII and transcribing with SP6.

$T R A A K$. A mouse clone of TRAAK (obtained from A. T. Gray) corresponding to nucleotides 266-1480 of the published sequence (GenBank accession number AF056492; Fink et al., 1998) was ligated into pcDNA3 using EcoRI and XhoI, linearized using HindIII, and transcribed using SP6.

TWIK-1. The coding region for human TWIK-1 [obtained from M. Lazdunski (Institut de Pharmacologie Moléculaire et Cellulaire, Valbonne, France); GenBank accession number NM002245; Lesage et al.,
1996] was subcloned into pcDNA3 using EcoRI and XhoI and linearized with EcoRI for transcription with SP6.

\section{Control experiments}

A number of control experiments were performed to establish the specificity of labeling. These included the use of corresponding sense probes (for TASK-1, TASK-2, TASK-3, and TREK-2) and separate experiments using specific oligonucleotide probes (for TASK-1, TREK-1, TRAAK, and TW IK-1). Hybridization with sense probes gave low levels of nonspecific (background) labeling, as expected. Hybridization with oligonucleotide probes resulted in the same set of distribution patterns as the corresponding cRNA probes. The oligonucleotides were as follows: TASK-1, cgcccaccagcaggtaggtgaaggtgcacacga, cacgaggttgaggaaggcgecgatgaccgtgag, and gagtactgcagcttctcgcggctcttgtaccag (nucleotides $170-138,829-797$, and 1123-1095 of the cloned rat TASK-1; GenBank accession number AF031384; Leonoudakis et al., 1998); TREK-1, cacaatggtggtcctctgggaaatctcctgagg and ggagagcttccgcttcacggatgtggcacgctg (nucleotides 726-694 and 1536-1504 of the cloned mouse TREK-1; GenBank accession number U73488.2; Fink et al., 1996); TRAAK, gaaatcctccaggctcttctggctcacacaggg and ctacaccggcacggccttgtctcggagtcgccc (nucleotides 466-434 and 1480-1448 of the cloned mouse TRAAK; GenBank accession number AF056492; Fink et al., 1998); and TWIK-1, cccaggccgatggtgctcagggagatgaaacag and ggccacaaaaggctcgttttgcttctgctcctc (nucleotides 731-699 and 1014-982 of the cloned rat TWIK-1; GenBank accession number AF022819).

To further rule out the possibility of spurious cross-reactivity of the cRNA probes, higher wash stringencies (i.e., final 30 min washes in $0.1 \times$ SSC and $25 \%$ formamide, at $55^{\circ} \mathrm{C}, 65^{\circ} \mathrm{C}$, and $75^{\circ} \mathrm{C}$ ) were used for control sagittal and horizontal sections. For all probes, these higher-stringency washes resulted in diminished hybridization, but the distribution of labeling was unchanged, indicating that for each probe the same mRNA species was labeled in the various brain regions.

\section{Data analysis and presentation}

All slides were exposed to film (Hyperfilm $\beta$-MAX; Amersham Pharmacia Biotech, Arlington Heights, IL) for the same length of time (5 d). The resulting autoradiograms were mounted on a light box and imaged using a video camera (CCD-72; Dage-MTI, Michigan City, IN). The same camera settings were used to generate images from each of the different genes. For resolution of cellular labeling, slides also were dipped in liquid autoradiographic emulsion (NTB2; Eastman Kodak, Rochester, NY), exposed for 5 weeks, and examined by low-power dark-field and high-power bright-field microscopy. Images of silver grains from these slides were captured using the same video camera mounted on a Leitz (Wetzlar, Germany) Diaplan microscope.

Pseudocolor images were rendered using image analysis software (MCID; Imaging Research Inc., St. Catharines, Ontario, Canada) to judge differential intensity of labeling of autoradiograms. Visual inspection of emulsion-dipped slides also was used to identify particular cell types and brain nuclei. Definitions for brain regions and nuclei were established following brain maps and identifying criteria from Paxinos and Watson (Paxinos, 1995; Paxinos and Watson, 1997). A combination of analysis of autoradiograms and emulsion-dipped slides was used to establish a comparative distribution of the transcripts, which is presented as a system of pluses (Table 1$)$, with five pluses $(+++++)$ indicating maximal signal intensity.

\section{RESULTS}

\section{Overview}

In situ hybridization was performed on brains and spinal cords using $\left[{ }^{33} \mathrm{P}\right]$-labeled cRNA probes specific for seven of the mammalian two-pore-domain $\mathrm{K}^{+}$channels. In initial experiments characterizing the probes, horizontal and sagittal brain sections (as well as transverse sections through the spinal cord) were used from two different rat strains, as well as from mice (see Materials and Methods). Transverse sections were taken through the neuraxis from adult Wistar rats; these were used for the figures presented in this report. Information regarding distribution also was verified using horizontal and sagittal sections.

In addition to the brain and spinal cord, sections were taken from dorsal root ganglia, in which all seven of the genes were 
Table 1. Distribution of two-pore-domain $\mathrm{K}^{+}$channel mRNA expression in the CNS

TASK-1 TASK-3

TREK-1

$$
++
$$$$
+++
$$

$+/+$

$++++$

Locus ceruleus

Midbrain and cerebellum

Superior/inferior colliculus

Periaqueductal gray

Red nucleus

Pontine nuclei

Interpeduncular nucleus

Vestibular nuclei

Cerebellar granule cell layer

Hypothalamus

Preoptic nuclei

Suprachiasmatic nucleus

Supraoptic nucleus

Lateral hypothalamic area

Paraventricular nucleus

Arcuate nucleus

Dorsomedial/ventromedial nuclei

Mammillary nuclei

Thalamus

Principal relay nuclei

Intralaminar nuclei

Reticular nucleus

Habenula

Basal forebrain

Striatum/accumbens

Olfactory tubercle

Globus pallidus

Subthalamic nucleus

Bed nucleus of the stria terminalis

Septum/diagonal band

Amygdala

Cortical nuclei

Nucleus of the lateral olfactory tract

Central nucleus

Medial nucleus

Lateral nucleus

Basolateral/basomedial nuclei

Hippocampus

Pyramidal cell layer, CA1

Pyramidal cell layer, CA2

Pyramidal cell layer, CA3

Granule cell layer, dentate gyrus

Indusium griseum

Cortex

Neocortex, layers II/III

Neocortex, layer IV

Neocortex, layer V

Neocortex, layer VI

Piriform cortex

Claustrum

Olfactory system

Olfactory bulb, glomerular layer

Olfactory bulb, granule cell layer

Anterior olfactory nucleus
$+++$

$++$

$+/++$

-
+++

$++$

$+1$

$+1-$
$+1-$

$+1-$

$++$

$++$

$+1-$

$+++$

$+1++$

$+/+$

$+/++$

$+/+$

$+$

$++1++$

$+/+$

$+/+$

$+1++$

$+1++$

$+/+$

$+$

$+$

$+1++$

$++$

$+/+$

$+1++$

$+/++$

$+1++$

-
$+/+$

$-$

$+/++$

$+1++$

$+/+$

$+1++$

$+1-$

+
$+/++$

$+/++$

$+/++$

$+/++$

$+/+$

$+/++$

$++/++$

$+/+$
$+1++$

$+++$

$-$

$++++$

$+/++$

$++/+++$

$++$

$++$

$++$

$+$

$++++$

$++$

$++++$

$+$

$+/+$

$++$

$+++$

$++/++++$

$+++1+++$

$++/++$

$+/+$

$+1-$

$+/+$

$-1+++$

$-1+++$

$+/+$

$++$

$+++$

$+1+$

$+1+++$

$+++$

$+$

$++1++++$

+
+

\section{$++$}

+
$-1++$

$+/+++$

$+$

$++1++$

$++$

$++$

$++$

$+++$

$++/++$

$+1++$

$++1++$

$++1++$

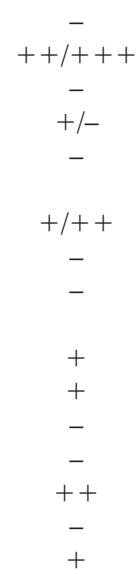

$++/++$

$-$

$+/+$

$+$

$++$

$+/+$

$+1-$

$-$

$+1-$

$+/++$

$-$

$+++/++++$

$+++/+++$

$-$

$+/++$

$-$

$+1++$

$+++$

$+$

$+/++$

$+1-$

$-$

$+1++$

$++1++$

$-$

$+/++$

$$
++
$$$$
+\stackrel{-}{+}++
$$$$
+
$$$$
+
$$

$++/+++$

$$
-
$$

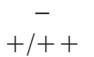

$-1+++$
TREK-2

TRAAK

TW IK-1

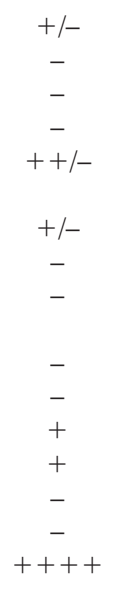

$+1-$
-
-
-
-

+ -

-
++

$+$

$+$

$-$

$+1-$

$+1-$

$++$

$++$

$+1++$

$+++$

+
$+/++$
+
-
$+/-$
$+/-$
$+/++$
+

$+/++$

$+/+$

$+/++$

$+$

+
$+/-$

$-$

$+1++$

$++\mathrm{H}+++$

$+1+++$

$-$

+
+

$+$

$+$

$+1++$

$+1++$

$++$

$-$

$+$

$+1++$

$+1++$
$+/-$

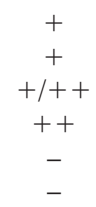

$-$

$+1+++$

$+$

$-$

$+$

$+/+$

$-1+$

$-1+$

$+/+$

$+$

$-$

$-1+$

$-1+$

$-1+$

$-1+$

$+1++$

$-$

$++$

$++$

$++$

$++$

$++$

$+$

$-1+$

$+1++$

$+1++$

$++$

$+$

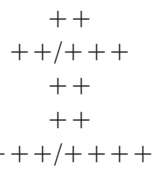

$++/++$

$++$

$++$

$++$

$+1++$

$+++1+++$

$+1++$

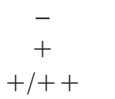

$+1++$

$+/+$

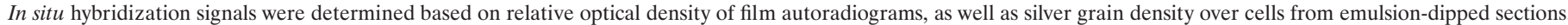

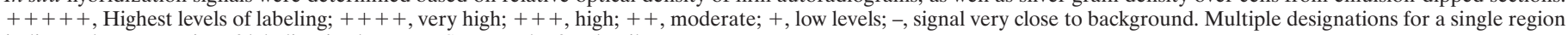
indicates heterogeneity of labeling in that area. See Results for details. 


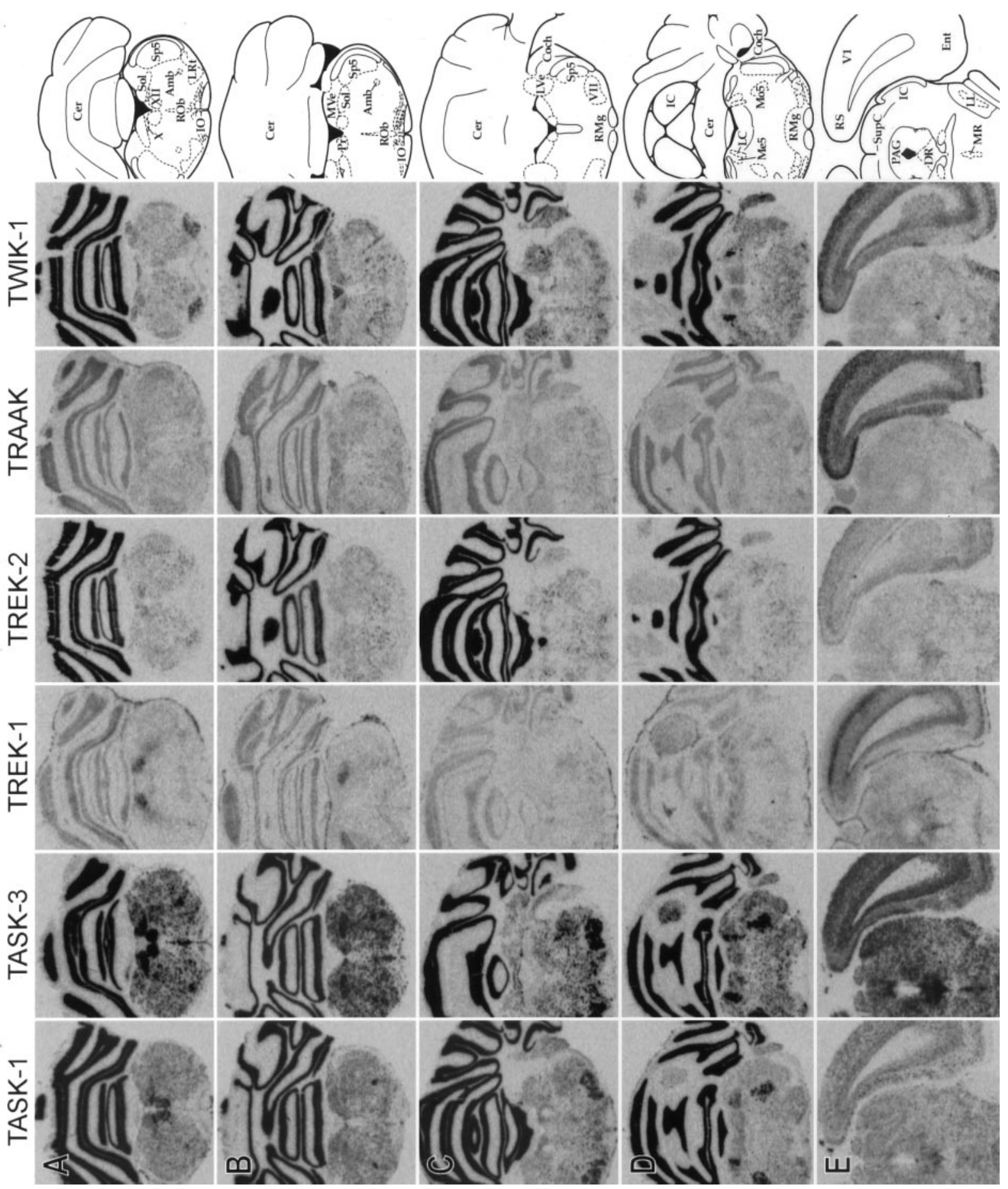

Figure 1. CNS distribution of two-pore-domain channels. Coronal sections were hybridized with $\left[{ }^{33} \mathrm{P}\right]-$ labeled cRNA probes for TASK-1, TASK-3, TREK-1, TREK-2, TRAAK, and TWIK-1 and exposed to autoradiographic film. Labeling for TASK-2 in the CNS was uniformly low and therefore is not presented here. Line drawings on the far right are adapted from Paxinos and Watson (1997; reproduced with permission) and indicate the relevant labeled areas. For abbreviations, please refer to Table 2. Scale bar, $3 \mathrm{~mm}$.

expressed (see below). However, only six of the transcripts displayed differential distribution in the CNS. The exception was TASK-2, for which labeling was uniformly low, although the intensity was a little higher than the corresponding sense probe. Because of the low signal and unremarkable pattern of localization, images of TASK- 2 in the CNS are not presented.

The relative intensities of labeling of the other six transcripts in the CNS were scored (see Materials and Methods) and are presented in Table 1. Note that the scoring system and descriptions of the results are based on a comparison of the relative signal intensities not only between different brain regions but also between the various probes. However, it should be understood that the ability to compare levels of different mRNA species (as opposed to levels of the same mRNA in different brain regions) is limited, because there are a number of factors besides cellular mRNA accumulation that can affect hybridiza- 


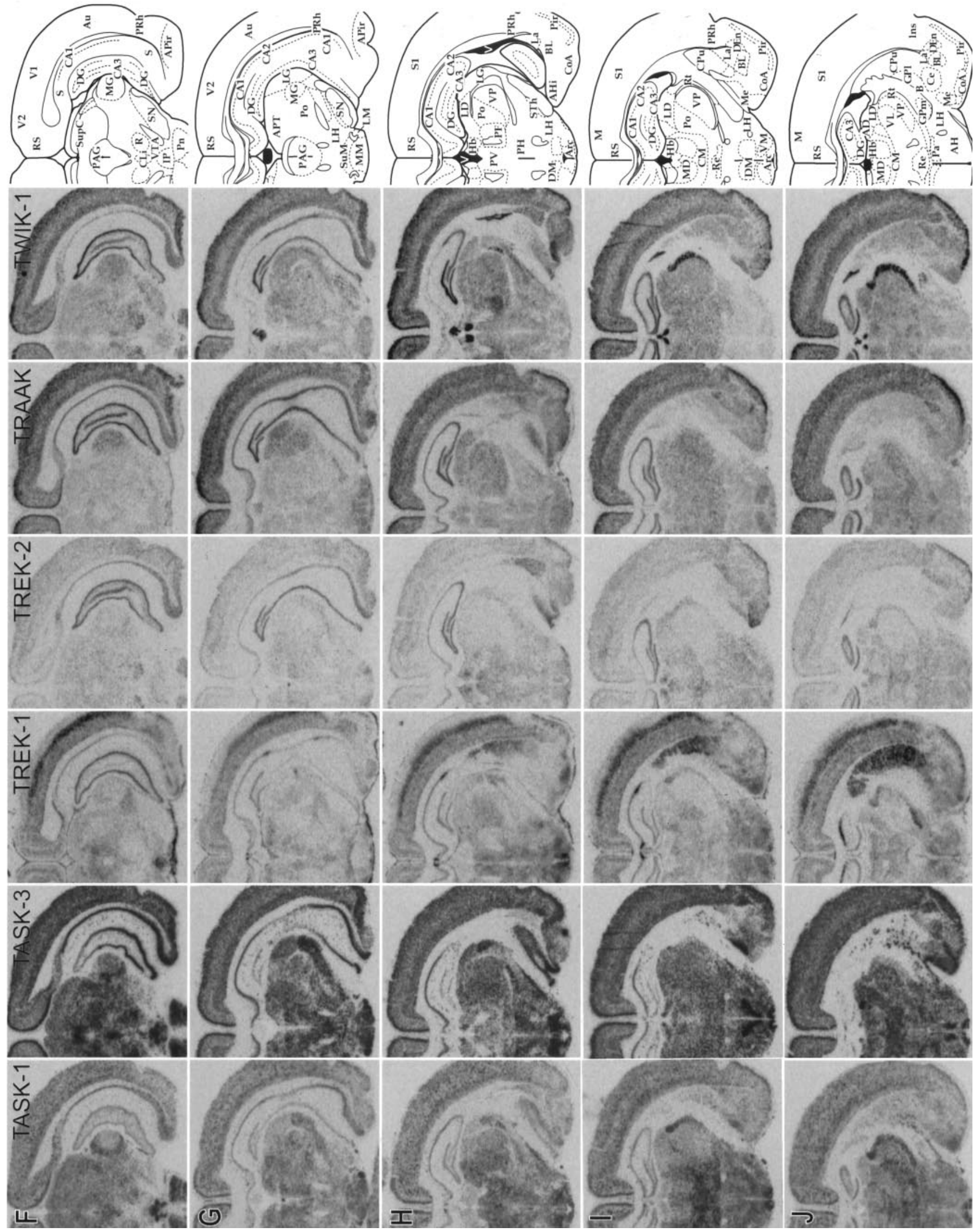




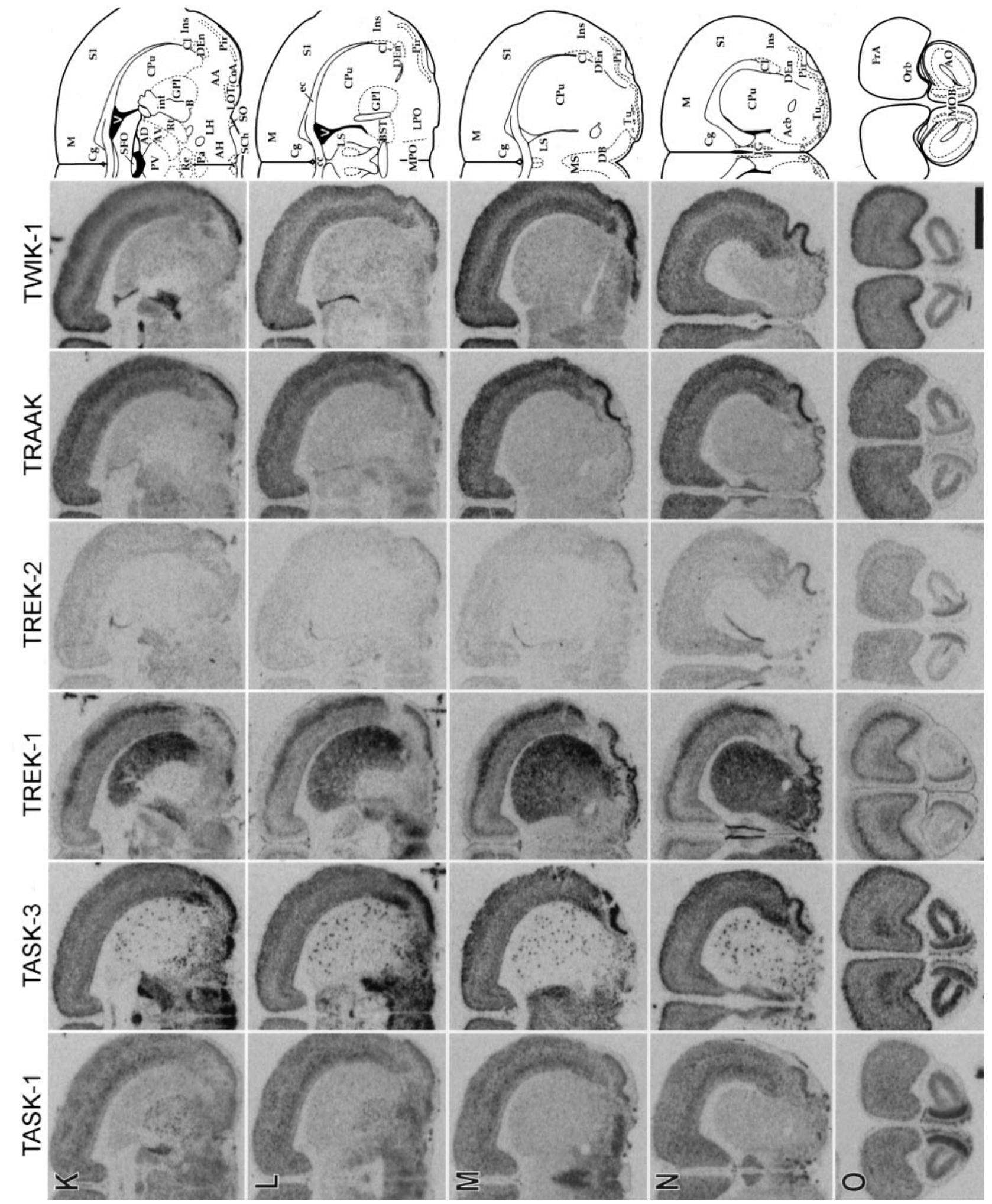

Figure 1. Continued

tion levels, such as the relative hybridization efficiencies of the different probes. Therefore, the results presented here reflect a comparison of mRNA accumulation in different brain regions for each gene considered separately; comparison of signal intensity generated by different probes should be viewed with caution.

\section{Non-neuronal expression}

For all six of the transcripts expressed in the CNS, labeling was consistent with neuronal expression, with the exception of a few regions. This non-neuronal labeling included a number of ventricular structures, such as the choroid plexus $(C h P)$, which 


\begin{tabular}{|c|c|c|c|}
\hline VII & Facial nucleus & $\mathrm{LM}$ & Lateral mammillary nucleus \\
\hline $\mathrm{X}$ & Dorsal motor nucleus & LOT & Nucleus of the lateral olfactory tract \\
\hline XII & Hypoglossal nucleus & LPO & Lateral preoptic area \\
\hline AA & Anterior amygdala & LRt & Lateral reticular nucleus \\
\hline Acb & Accumbens nucleus & LS & Lateral septal nucleus \\
\hline $\mathrm{AD}$ & Anterodorsal thalamic nucleus & LVe & Lateral vestibular nucleus \\
\hline $\mathrm{AH}$ & Anterior hypothalamic area & M & Motor cortex \\
\hline $\mathrm{AHi}$ & Amygdalohippocampal area & MD & Mediodorsal thalamic nucleus \\
\hline Amb & Nucleus ambiguous & Me5 & Mesencephalic trigeminal nucleus \\
\hline $\mathrm{AO}$ & Anterior olfactory nucleus & $\mathrm{Me}$ & Medial amygdaloid nucleus \\
\hline APir & Amygdalopiriform transition area & MG & Medial geniculate nucleus \\
\hline APT & Anterior pretectal nucleus & MM & Medial mammillary nucleus \\
\hline Arc & Arcuate nucleus & Mo5 & Motor trigeminal nucleus \\
\hline $\mathrm{Au}$ & Auditory cortex & MOB & Main olfactory bulb \\
\hline AV & Anteroventral thalamic nucleus & MPO & Medial preoptic nucleus \\
\hline B & Basal nucleus & MR & Median raphe nucleus \\
\hline $\mathrm{BL}$ & Basolateral amygdaloid nucleus & MS & Medial septal nucleus \\
\hline BST & Bed nucleus of the stria terminalis & $\mathrm{MVe}$ & Medial vestibular nucleus \\
\hline CA1 & Field CA1 of hippocampus & Orb & Orbital cortex \\
\hline CA2 & Field CA2 of hippocampus & $\mathrm{Pa}$ & Paraventricular hypothalamic nucleus \\
\hline CA3 & Field CA3 of hippocampus & PAG & Periaqueductal gray \\
\hline $\mathrm{cc}$ & Corpus callosum & PF & Parafascicular thalamic nucleus \\
\hline $\mathrm{Ce}$ & Central amygdaloid nucleus & $\mathrm{PH}$ & Posterior hypothalamic area \\
\hline Cer & Cerebellum & Pir & Piriform cortex \\
\hline $\mathrm{Cg}$ & Cingulate cortex & PMCo & Posteromedial cortical amygdaloid nucleus \\
\hline $\mathrm{ChP}$ & Choroid plexus & Pn & Pontine nuclei \\
\hline $\mathrm{Cl}$ & Claustrum & Po & Posterior thalamic nuclear group \\
\hline CLi & Caudal linear nucleus of the raphe & $\operatorname{Pr}$ & Propositus nucleus \\
\hline $\mathrm{CM}$ & Central medial thalamic nucleus & $\mathrm{PRh}$ & Perirhinal cortex \\
\hline $\mathrm{CoA}$ & Cortical amygdaloid nuclei & PV & Paraventricular thalamic nucleus \\
\hline Coch & Cochlear nuclei & $\mathrm{R}$ & Red nucleus \\
\hline $\mathrm{CPu}$ & Caudate putamen (striatum) & $\mathrm{Re}$ & Reuniens thalamic nucleus \\
\hline DB & Diagonal band & $\mathrm{RMg}$ & Raphe magnus nucleus \\
\hline DEn & Dorsal endopiriform nucleus & $\mathrm{ROb}$ & Raphe obscurus nucleus \\
\hline DG & Dentate gyrus & RS & Retrosplenial cortex \\
\hline $\mathrm{DM}$ & Dorsomedial hypothalamic nucleus & $\mathrm{Rt}$ & Reticular thalamic nucleus \\
\hline DR & Dorsal raphe nucleus & $\mathrm{S}$ & Subiculum \\
\hline ec & External capsule & $\mathrm{S} 1$ & Primary somatosensory cortex \\
\hline Ent & Entorhinal cortex & $\mathrm{SCh}$ & Suprachiasmatic nucleus \\
\hline f & Fornix & SFO & Subfornical organ \\
\hline FrA & Frontal association cortex & $\mathrm{SN}$ & Substantia nigra \\
\hline GPl & Lateral globus pallidus & $\mathrm{SO}$ & Supraoptic nucleus \\
\hline GPm & Medial globus pallidus & Sol & Nucleus of the solitary tract \\
\hline $\mathrm{Hb}$ & Habenula & Sp5 & Spinal trigeminal nucleus \\
\hline IC & Inferior colliculus & STh & Subthalamic nucleus \\
\hline IG & Indusium griseum & $\mathrm{SuM}$ & Supramammillary nucleus \\
\hline Ins & Insular cortex & SupC & Superior colliculus \\
\hline int & Internal capsule & $\mathrm{Tu}$ & Olfactory tubercle \\
\hline $\mathrm{IO}$ & Inferior olivary nucleus & $\mathrm{V}$ & Ventricle \\
\hline IP & Interpeduncular nucleus & V1 & Primary visual cortex \\
\hline $\mathrm{La}$ & Lateral amygadloid nucleus & $\mathrm{V} 2$ & Secondary visual cortex \\
\hline $\mathrm{LC}$ & Locus ceruleus & VL & Ventrolateral thalamic nucleus \\
\hline LD & Laterodorsal thalamic nucleus & $\mathrm{VM}$ & Ventromedial thalamic nucleus \\
\hline LG & Lateral geniculate nuclei & VP & Ventral posterior thalamic nucleus \\
\hline LH & Lateral hypothalamic area & VTA & Ventral tegmental area \\
\hline LL & Nuclei of the lateral lemniscus & & \\
\hline
\end{tabular}



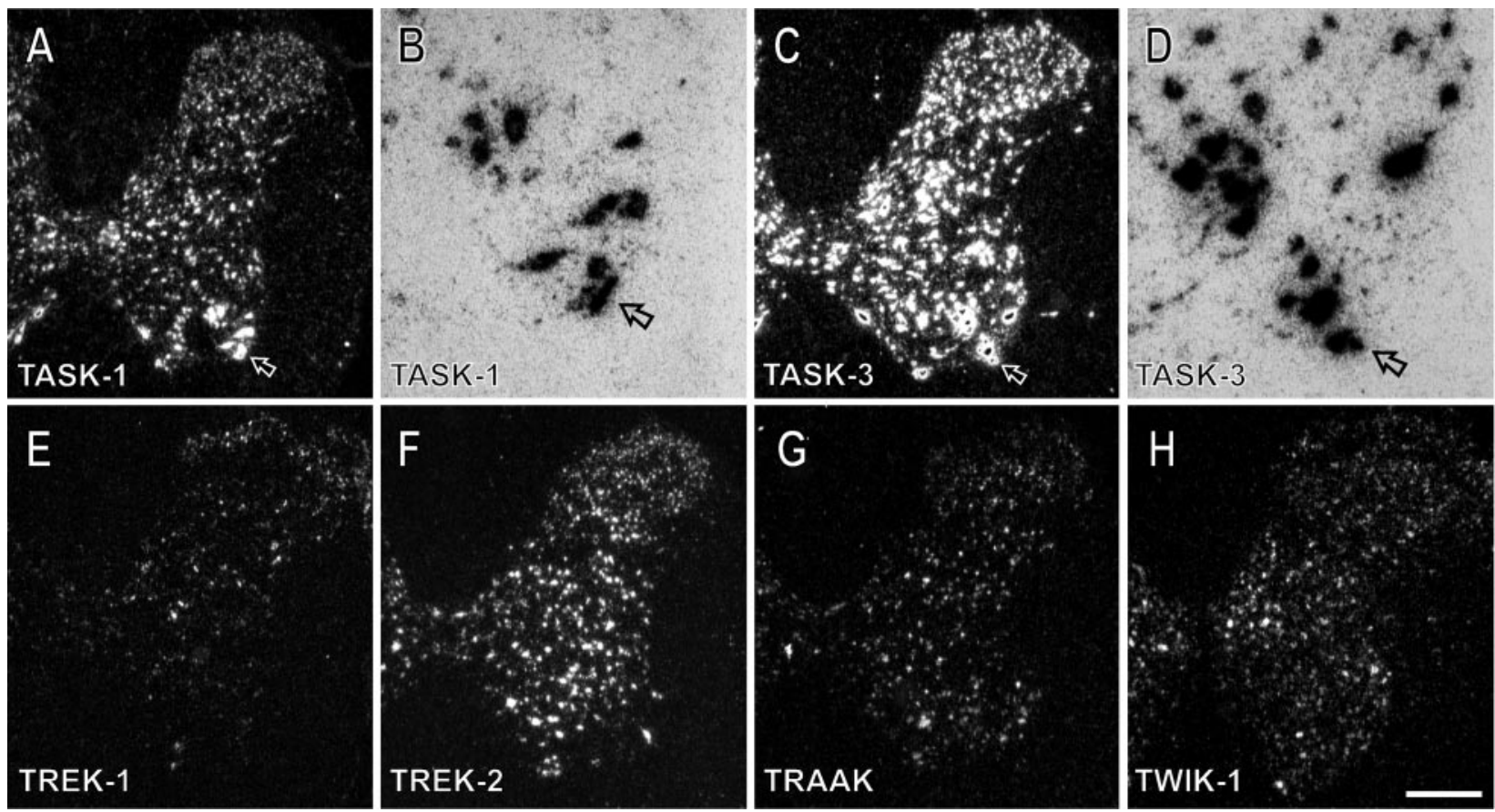

Figure 2. Spinal cord expression of two-pore-domain $\mathrm{K}^{+}$channel transcripts. Transverse sections from rat cervical spinal cord were hybridized with $\left[{ }^{33} \mathrm{P}\right]$-labeled cRNA probes and subsequently dipped in liquid autoradiographic emulsion. Silver grains were imaged using dark-field and bright-field microscopy. Top panels $(A-D)$ show broad distribution of TASK-1 and TASK-3, with low-to-moderate accumulations of silver grains over many neurons Ventral horn motoneurons were intensely labeled for both transcripts. In the alternate panels $(B, D)$, these same motoneurons are shown at higher power using bright-field optics, with the corresponding cells indicated by arrows. Bottom panels $(E-H)$ show dark-field images of labeling for the other transcripts in the spinal cord. Labeling for these transcripts was lower, although there were moderate levels of TREK-2 in spinal cord interneuron layers (F). Scale bar: $A, C, E-H, 400 \mu \mathrm{m} ; B, D, 100 \mu \mathrm{m}$.
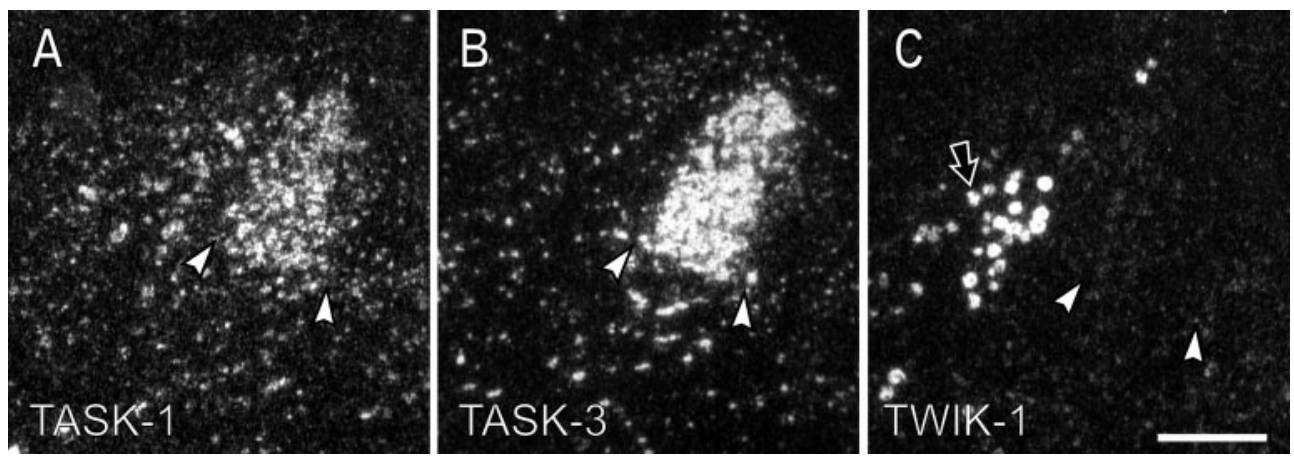

Figure 3. TASK-1 and TASK-3 show high levels of mRNA accumulation in the locus ceruleus; TWIK-1 is highly expressed by adjacent mesencephalic trigeminal neurons. Panels show dark-field images of coronal sections through the dorsolateral pons corresponding to autoradiograms in Figure $1 D$. Arrowheads in each panel mark the approximate boundary of the locus ceruleus, which had high levels of labeling for TASK-1 $(A)$ and was even more densely labeled for TASK-3 $(B)$. Large mesencephalic trigeminal neurons are located lateral to the locus ceruleus; these neurons showed high levels of TWIK-1 (arrow in $C$ ). Scale bar, $300 \mu \mathrm{m}$.

expressed high levels of TWIK-1 (e.g., Fig. $1 H, K, L$; see Fig. 4G), the subfornical organ $(S F O)$, which expressed high levels of TASK-3 (Fig. $1 K$ ), and the ependymal cells lining the ventricles, which rostrally expressed moderate-to-high levels of TREK-2 (e.g., Fig. $1 N$ ) (see Table 2 for a complete list of all abbreviations used). In addition, the pia mater lining the brain contained message for TREK-1; this labeling could be seen in a number of sections (e.g., Fig. $1 A, G$, at the edge of sections) where the pia mater had been left intact during tissue preparation. Aside from these structures, labeling was present in areas rich in neuronal cell bodies but absent in fiber tracts in which only glial labeling is expected (e.g., Fig. 1L, ec, int, $c c$; see Fig. 4).

\section{Medulla and spinal cord}

The medulla and spinal cord were characterized by widespread expression of TASK-3, moderate expression of TASK-1, and generally low expression (with some notable exceptions) of the other transcripts. This was the case in primary sensory areas of the spinal cord and medulla (Figs. $1 A-C, S p 5$ in the medulla, 2, dorsal horn of the spinal cord), where all transcripts were low except for TASK-1 and TASK-3, which were moderate. Cells of the cochlear nuclei (Coch) expressed moderate levels of TASK-1, TASK-3, and TWIK-1 (Fig. $1 C, D$ ). The nucleus of the solitary tract $(\mathrm{Sol})$, which is associated with visceral sensory afferents, had 

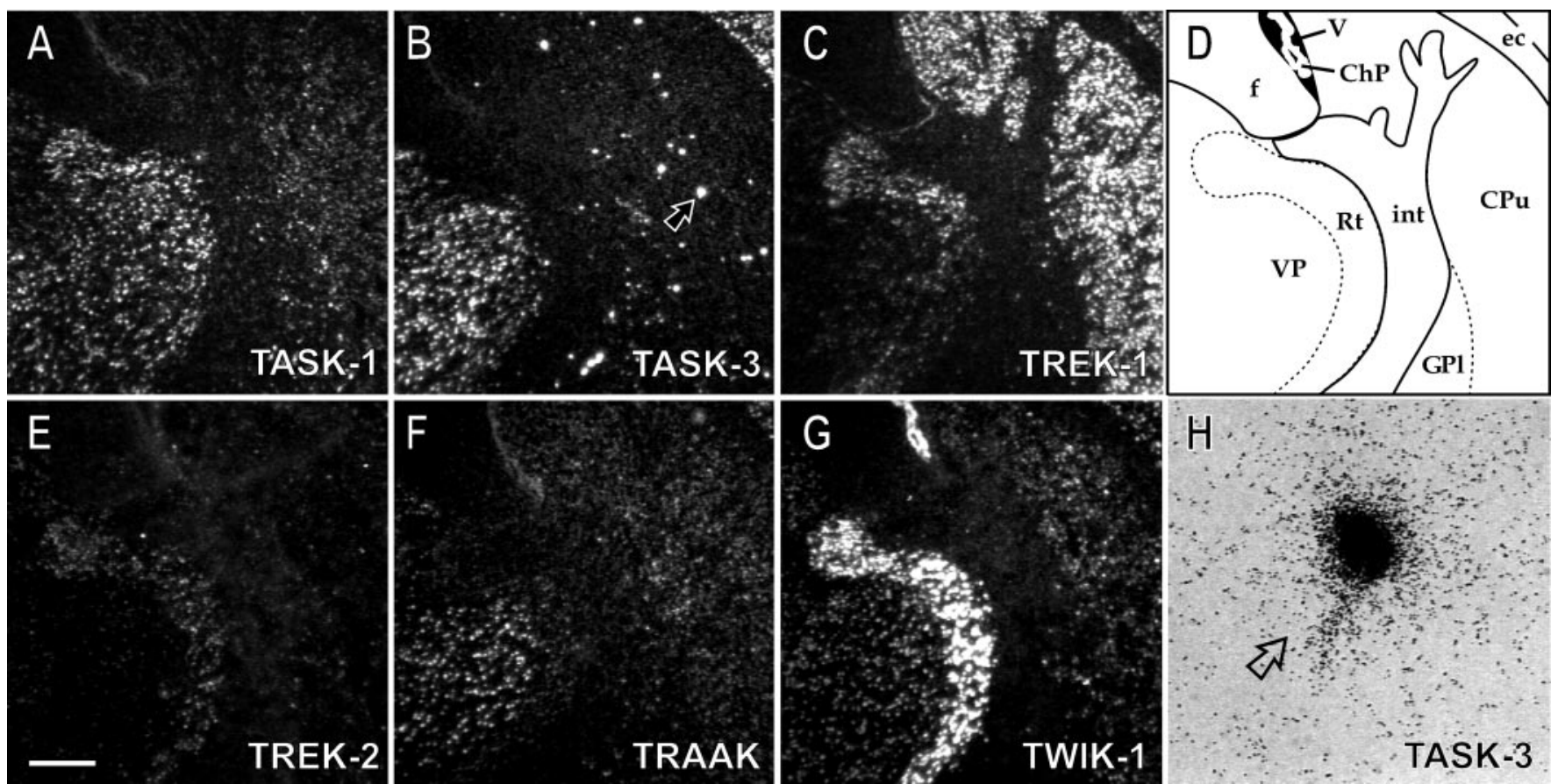

Figure 4. Expression of two-pore-domain channels in the thalamus and striatum. Shown are low-power dark-field images of emulsion-dipped sections through the telencephalon, corresponding to the film autoradiograms in Figure $1 J$. The line drawing $(D)$ indicates the relevant fiber tracts and brain nuclei (for abbreviations, see Table 2). In the caudate putamen, there was uniformly high signal for TREK-1 in cells densely distributed throughout the nucleus $(C)$; these are likely GABAergic output neurons. In contrast, TASK-3 labeled a set of large, sparsely located cells, likely cholinergic interneurons, one of which is marked with an arrow in the dark-field image $(B)$ and in the corresponding high-power bright-field micrograph in $H$. Also note the intense signal for TWIK-1 in the thalamic reticular nucleus and in the choroid plexus (G). Scale bar: $A-G, 500 \mu \mathrm{m} ; H, 25 \mu \mathrm{m}$.

moderate-to-high levels of both TREK-1 and TASK-3 (Fig. $1 A, B)$.

Somatic motoneurons expressed high levels of TASK-1 and TASK-3 (Figs. $1 A, C, D, 2$ ). Whereas TASK-3 labeling was uniformly strong, TASK-1 was highest in the motor trigeminal (Mo5) and in select neurons of the spinal cord (see also Talley et al., 2000). Autonomic motoneurons in the dorsal motor nucleus $(X)$ and nucleus ambiguous $(\mathrm{Amb})$ also showed high levels of TASK-1 and TASK-3 (Fig. 1A,B).

Neurons in the reticular fields of the medulla and pons (Fig. $1 A-E$ ), as well as interneuronal populations of the spinal cord (Fig. 2), contained moderate-to-high levels of mRNA for TASK-3 and moderate levels of TASK-1 but lower levels of the other transcripts. TREK-2 moderately labeled scattered neurons in this region (particularly in the spinal cord) (Fig. 2), as did TWIK-1. This latter transcript was expressed at moderate-to-high levels in the lateral reticular nucleus (Fig. 1A, $L R t$ ). Neurons of the inferior olive (Fig. 1A, $B, I O$ ) did not express appreciable levels of any of the transcripts.

\section{Brainstem aminergic nuclei}

Raphe nuclei, which contain serotonergic neuronal cell bodies, showed particularly high expression of TASK-3. This was evident in both the pons (Fig. $1 E, D R, M R$ ) and the medulla (Fig. $1 A, B$, $R O b, R M g$ ). Low-to-moderate levels of TASK-1 were found in these regions; this was also the case for TREK-1 in the dorsal raphe nucleus $(D R)$. The locus ceruleus $(L C)$, which contains noradrenergic cell bodies, also was characterized by high levels of TASK-3 and TASK-1 mRNA (Figs. $1 D, 3$ ). Note that this nucleus is flanked laterally by mesencephalic trigeminal $(\mathrm{Me} 5)$ neurons, which expressed high levels of TWIK-1 (Figs. 1D, 3; see below). In contrast to the raphe nuclei and the locus ceruleus, dopami- nergic neurons of the substantia nigra $(S N)$ were virtually unlabeled by these transcripts, with the exception of very low levels of TASK-3 and TWIK-1 (Fig. $1 F, G$ ). The ventral tegmental area (VTA), which also contains dopaminergic neurons, showed moderate labeling for TASK-3 (Fig. $1 F$ ).

\section{Midbrain and cerebellum}

Other regions of the midbrain also were characterized by substantial levels of TASK-3 mRNA accumulation. These included the inferior colliculus (Fig. $1 D, E, I C$ ), the red nucleus, $R$, which also had low-to-moderate levels of TWIK-1 and TREK-2) (Fig. $1 F$ ), and the pontine nuclei, $P n$, which also had high levels of TASK-1 and moderate levels of TWIK-1) (Fig. $1 F$ ). There was very high labeling for TASK- 3 in the periaqueductal gray $(P A G)$; this was particularly evident in the dorsal region (Fig. 1E,F). The interpeduncular nucleus $(I P)$ was labeled by high levels of both TASK-1 and TASK-3, as well as moderate levels of TREK-1 (Fig. $1 F$ ). Two midbrain cell groups were notable for their high levels of expression of TWIK-1. One was the mesencephalic trigeminal nucleus (Me5) (Figs. 1D, 3; noted above). This nucleus contains very large cell bodies of primary afferent neurons, which are similar in function to neurons of the dorsal root ganglia. Notably, some of the very large cells in the dorsal root ganglia also expressed high levels of TW IK-1 (see below). The other midbrain region expressing high levels of TWIK-1 was the superior olivary complex (data not shown).

In contrast to the marked differential expression of the CNS two-pore-domain channel transcripts in most brain regions, the granule cell layer of the cerebellum $(\mathrm{Cer})$ was characterized by expression of all six genes (Fig. 1A-E). This expression was extremely high for TASK-1, TASK-3, TREK-2, and TWIK-1 and lower but still appreciable for TREK-1 and TRAAK. TWIK-1 
also was expressed in Purkinje cells and in neurons of the deep cerebellar nuclei (data not shown); this latter expression was similar to that of the adjacent vestibular nuclei (Fig. $1 C, L V e$ ).

\section{Hypothalamus}

The hypothalamus had extremely high levels of TASK-3 in certain nuclei, along with high levels of TASK-1 and TREK-1. Labeling for TASK-3 was particularly intense in the dorsomedial nucleus $(D M)$ and the arcuate nucleus $(A r c)$ (Fig. $1 H, I)$, the suprachiasmatic nucleus (Fig. $1 K, S C h$ ), and lateral portions of the mammillary bodies (Fig. $1 G, L M$ ). It also was high, but less dramatically so, in the medial portion of the mammillary bodies (Fig. $1 G, M M$ ), as well as in the medial preoptic nucleus (Fig. $1 L$, $M P O)$. Moderate levels were seen in the lateral hypothalamic (Fig. $1 G-K, L H$ ) and lateral preoptic areas (Fig. $1 L, L P O)$ and in the ventromedial (Fig. 1I,VM) and paraventricular (Fig. $1 J, K$, $\mathrm{Pa}$ ) nuclei. TASK-1 had a similar distribution to TASK-3: highest in the dorsomedial and arcuate nuclei (Fig. $1 H, I$ ); in the lateral portions of the mammillary bodies (Fig. $1 G$ ), and in the medial preoptic nucleus (Fig. $1 L$ ), but at lower intensity levels. TREK-1 also was high in the medial preoptic nucleus (Fig. $1 L$ ) and the arcuate nucleus (Fig. $1 H, I)$ and moderate in the dorsomedial and ventromedial nuclei (Fig. 1H,I). Expression of both TWIK-1 and TREK-2 was low in the hypothalamus. TRAAK also was low, with the exception of moderate expression in the ventromedial nucleus (Fig. 1I) and suprachiasmatic nucleus (Fig. 1K).

\section{Thalamus}

In the thalamus, the principal relay nuclei had low-to-moderate levels of all of the transcripts except TASK-3. This transcript was moderate-to-high in most relay nuclei, including the medial geniculate $(M G)$, posterior $(P o)$, ventral posterior $(V P)$, laterodorsal $(L D)$, and anteroventral $(A V)$ nuclei (Figs. $1 F-K, 4)$. However, labeling for TASK-3 was high in the ventral portion of the lateral geniculate nucleus (Fig. $1 G, H, L G$ ) and was especially prominent in the anterodorsal nucleus (Fig. $1 J, K, A D$ ). TASK-1 was higher in the intralaminar thalamic nuclei, especially in the central medial and reuniens nuclei (Fig. 1I,J). TW IK-1 was found at very high levels in the thalamic reticular nucleus $(R t)$, which forms a shell of GABAergic interneurons surrounding the relay nuclei (Figs. $1 I-K, 4)$. In addition, this nucleus had moderate levels of TASK-1 and TREK-1 (Figs. $1 I-K, 4$ ). TWIK-1 also was extremely high in the medial habenula (Fig. $1 H-J$ ), although it should be noted that this labeling primarily was restricted to the ventral portion of the medial nucleus. Finally, there also was notable labeling for TWIK-1 in the parafascicular thalamic nucleus (Fig. $1 H, P F)$.

\section{Basal forebrain and amygdala}

The basal ganglia were characterized by high expression of TREK-1 and TASK-3; these two transcripts were distributed in the striatum $(\mathrm{CPu})$ in a manner consistent with two distinctive cell types (Figs. 1I-N, 4). TREK-1 was evenly expressed at high levels across the striatum. This dense and relatively uniform expression is consistent with localization in GABAergic output neurons, which constitute the vast majority of striatal neurons. In contrast, TASK-3 was expressed by larger, much more sparsely distributed cells, a characteristic of cholinergic striatal interneurons. For both TASK-3 and TREK-1, their respective distribution patterns extended into the accumbens nucleus $(A c b)$ and the olfactory tubercle $(T u)$ (Fig. 1M,N). Expression of the other transcripts in these regions was low,
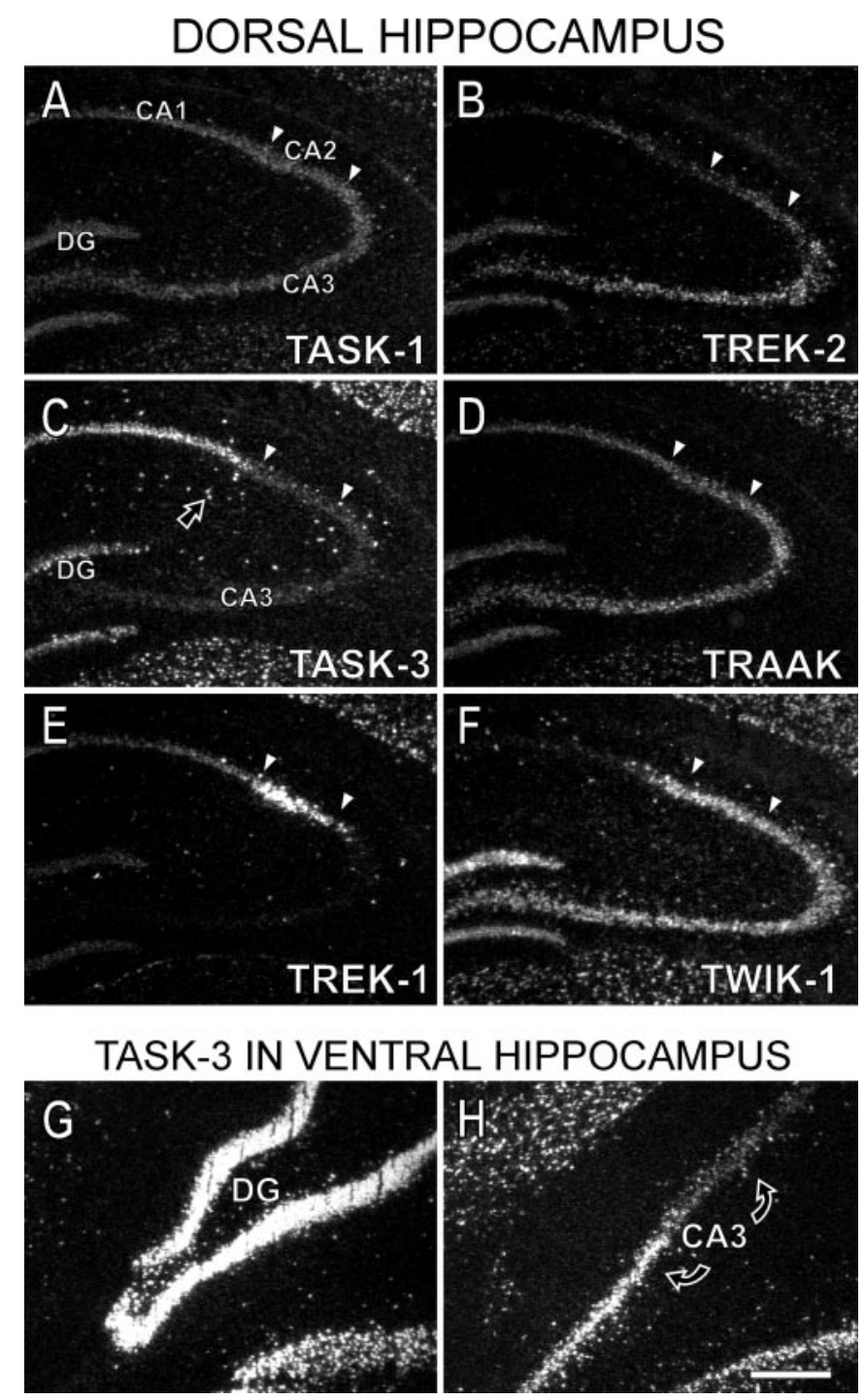

Figure 5. Expression of two-pore-domain channels in different regions of the hippocampus. Low-power dark-field images of emulsion-dipped coronal sections through the hippocampus. $A-F$ show labeling for the six channel mRNAs through the rostral/dorsal hippocampus; this view corresponds to the film autoradiograms seen in Figure $1 I$. Note the differential distribution through the various pyramidal cell populations $(C A 1$, $C A 2$, and $C A 3$ ), the approximate boundaries of which are marked by arrowheads. Also, there was TASK-3 labeling in scattered cells of the nonpyramidal layers (arrow in $C$ ); these are likely hippocampal interneurons. $G$ and $H$ illustrate differential TASK-3 expression in the ventral/ caudal hippocampus compared with the rostral/dorsal expression illustrated above. $G$ shows TASK-3 expression in the ventral/caudal dentate gyrus and corresponds to the ventral portion of the film autoradiogram in Figure $1 F$. Note that there was particularly intense labeling of dentate gyrus granule cells in the ventral hippocampus but very little expression by these cells in the dorsal hippocampus $(C)$. $H$ shows TASK-3 expression in the ventral/caudal portion of CA3 and is taken from a section corresponding to the film autoradiogram seen in Figure $1 G$. The CA3 pyramidal cell layer is marked by curved arrows and traverses the entire panel. Note that there is much more TASK-3 expression in cells in the bottom (i.e., ventral) part of this panel when compared with cells in the top part of the panel and when compared with TASK-3 labeling in CA3 of the dorsal hippocampus $(C)$. Scale bar, $500 \mu \mathrm{m}$.

with the exception of TASK-1 in the olfactory tubercle, where it was expressed at moderate-to-high levels in some of the cellular islands (Fig. $1 N$ ).

It should be noted that the scattering of TASK-3-labeled neu- 

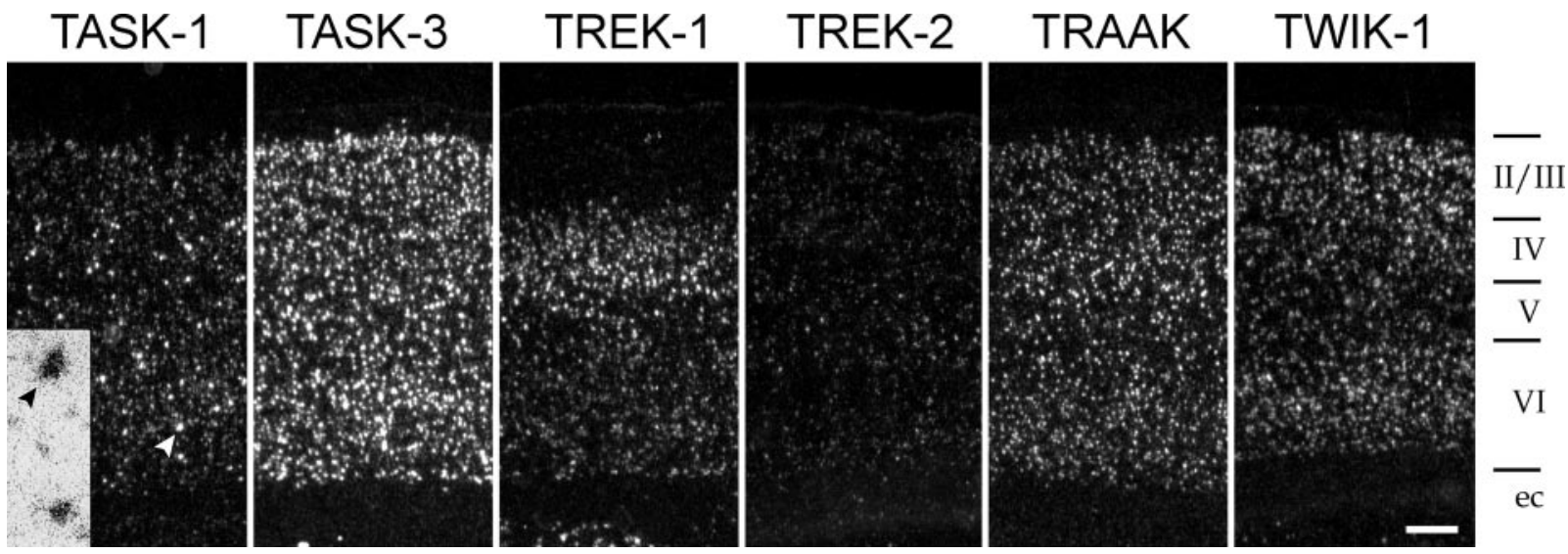

Figure 6. Laminar distribution of two-pore-domain $\mathrm{K}^{+}$channel transcripts in the neocortex. Shown are dark-field micrographs of the somatosensory cortex. The relevant cell body layers (i.e., layer II through layer VI) are indicated on the right, as is the underlying fiber tract [external capsule (ec)]. TASK-3, TRAAK, and TWIK-1 could be seen at appreciable levels in all layers, whereas TREK-1 was highly enriched in layer IV. TASK-1 was expressed at higher levels in a scattering of cells, mostly in layers IV and VI. One of these layer VI cells is marked by an arrowhead and is shown using high-power bright-field optics in the inset on the left. Scale bar: $250 \mu \mathrm{m}$; inset, $50 \mu \mathrm{m}$.

rons seen in the striatum extended into the region of the basal nucleus $(B)$, just lateral to the internal capsule (int), and in the medial portion of the lateral globus pallidus $(G P l)$ (Fig. $1 J, K)$. This pattern once again is consistent with TASK-3 expression in neurons of a cholinergic phenotype, because the basal nucleus contains a diffuse collection of large (cortically projecting) cholinergic neurons (Fig. $1 J, K$ ). TASK-1 labeling was present at low-to-moderate levels in the lateral globus pallidus (Fig. $1 J-L$ ), although in contrast to TASK-3, this labeling appeared to be uniform throughout the nucleus. Also with regard to the globus pallidus, TASK-3 and TW IK-1 were expressed at moderate levels in the medial segment (Fig. $1 J, G P m$ ). Finally, the subthalamic nucleus $(S T h)$, another nucleus of the basal ganglia, was characterized by high levels of TASK-3 and moderate levels of TASK-1 and TWIK-1 (Fig. 1H).

In the more medial nuclei of the basal forebrain, the bed nucleus of the stria terminalis (BST) contained cells expressing high levels of TASK-3 and low-to-moderate levels of TASK-1 and TREK-1 (Fig. 1L). There were moderate levels of TASK-3 in the lateral septum $(L S)$ and moderate levels of TASK-1 in the medial septum $(M S)$ and diagonal band $(D B)$ (Fig. $1 M)$.

In the amygdala, labeling was highest in the cortical nuclei situated along the ventrolateral surface of the forebrain (Fig. $1 F-K)$. Caudally, these included the amygdalopiriform transition area (Fig. $1 F, G, A P i r$ ) and the amygdalohippocampal area (Fig. $1 \mathrm{H}, \mathrm{AHi}$ ), both of which had high levels of TASK-3 and TWIK-1 and moderate levels of TASK-1, TREK-2, and TRAAK. TASK-3 and TWIK-1 also were high in the rostral cortical amygdaloid nuclei (Fig. $1 J, K, C o A$ ), whereas the other transcripts were more moderately expressed. The nucleus of the lateral olfactory tract $(L O T)$, which is at the rostral end of the amygdala (Fig. $1 K$ ), had high levels of TASK-3 and TREK-1 and moderate levels of TRAAK. The other area of note in the amygdala was the medial nucleus (Fig. 1I,J, Me). TASK-3, TREK-1, and TREK-2 all were more highly enriched in the posterior part of this nucleus, with TASK-3 at particularly high levels. There was low-to-moderate labeling throughout the nucleus for TASK-1, TRAAK, and TWIK-1.

\section{Hippocampus}

Hippocampal pyramidal neurons expressed all transcripts but with differences in distribution (Figs. $1 F-J, 5$ ). TASK-1 and
TRAAK were low-to-moderate, with relatively even levels of expression throughout. In contrast, TWIK-1 showed moderate labeling in CA2 and CA3 but low levels in CA1, particularly in the caudal sections (Fig. $1 F-H$ ). TREK-2 had lower levels of labeling but also showed more expression in CA3 than CA1.

For TASK-3, there was a distinct gradient of expression, with more expression ventrally, particularly in CA3 pyramidal neurons, where expression was moderate-to-high at the most ventral/ caudal (i.e., temporal) end of the hippocampus (Figs. $1 G, 5 H$ ) but nearly undetectable at the rostral/dorsal (i.e., septal) end (Figs. $1 H-J, 5 C)$. This TASK-3 expression gradient was even more dramatic for dentate gyrus $(D G)$ granule cells, which were intensely labeled ventrally (Figs. $1 F, 5 G$ ) but had only low densities of silver grains dorsally (Figs. $1 H-J, 5 C$ ).

The distribution of TREK-1 also was interesting; it was lowto-moderate in CA1 and almost absent in CA3 but quite high in CA2 and in a limited region of cells adjacent to the hippocampal commissure (Fig. 1G-I, the most medial portion of the hippocampus). These two sets of highly expressing cells were conjoined in the very rostral hippocampus, in a region identified as CA3 (Fig. $1 J)$. A potentially related set of cells also expressed high levels of TREK-1: neurons of the indusium griseum (Fig. $1 N, I G$ ). This structure has been hypothesized to be functionally related to the hippocampus (Paxinos, 1995).

There also was expression by cells (presumably interneurons) in nonpyramidal layers of the hippocampus and in the polymorph layer of the dentate gyrus. This was particularly notable for TASK-3 but also was evident for TASK-1, TREK-1, and TW IK-1 (Fig. 5). This labeling was not noticeably restricted to any particular hippocampal laminas.

\section{Cerebral cortex}

The neocortex was associated with high levels of TASK-3, TRAAK, TWIK-1, and TREK-1 (Figs. $1 E-N, 6$ ). In the case of these first three genes, there was labeling in all cell body layers. This was in contrast to TREK-1, in which high labeling was restricted to layer IV, and there was virtually no expression in layers II-III. The pattern for TREK-1 was consistent with expression in layer IV granule neurons, inasmuch as it was particularly evident in the primary visual and primary somatosensory cortices (Fig. 1: $E, F, V 1 ; H-N, S 1$ ), which contain a high density of this cell type. TREK-2 was low throughout, although it was somewhat 

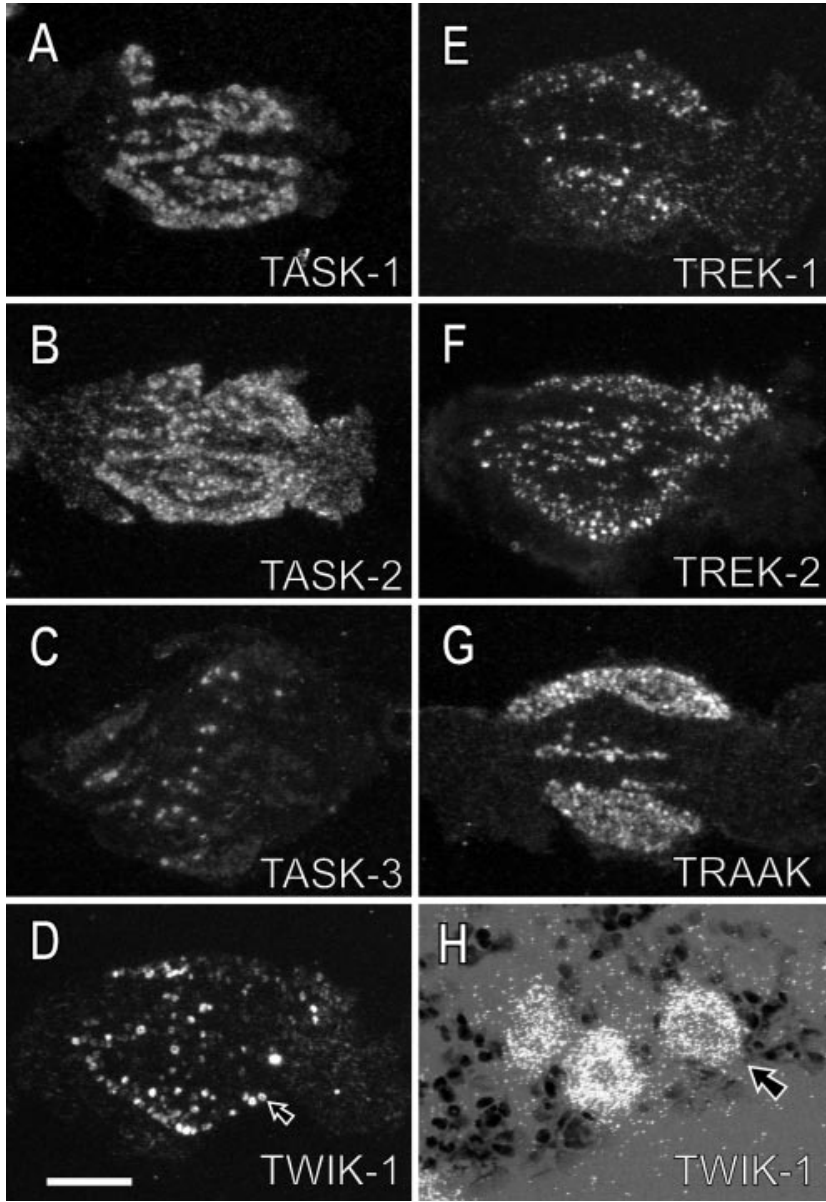

Figure 7. Two-pore-domain channel expression in dorsal root ganglia. As shown by dark-field microscopy, all seven transcripts were expressed in dorsal root ganglia, and especially high densities of silver grains were seen for TASK-1 $(A)$, TASK-2 $(B)$, and TRAAK $(G)$. TASK-3 $(C)$ was expressed at high levels but by a much more limited proportion of the cells. Labeling for TWIK-1 was for the most part restricted to larger neurons; it is shown at low power in $D$, with a higher-power view of the indicated neuron (arrows) shown in $H$. Scale bar: $A-G, 400 \mu \mathrm{m} ; H, 50 \mu \mathrm{m}$.

higher in the most caudal (Fig. 1E, V1, Ent) and rostral (Fig. 1O, FrA, Orb) portions of the cortex. This preferential rostral and caudal labeling also was evident in sagittal and horizontal sections (data not shown). TASK-1 was expressed at low-to-moderate levels throughout, but this lower expression was punctuated by a scattering of more heavily labeled cells, especially in layers IV and VI (Fig. 6, inset). Also, TASK-3 and TW IK-1 were expressed at higher levels in the outermost layers (II-III) in the motor cortex (Fig. $1 I-N, M$ ) and in the frontal association cortex (Fig. 1O).

The allocortex, including cortices on the midline (Fig. 1: $E-J$, $R S ; K-N, C G$ ) and structures ventral to the neocortex (Fig. 1: $F-I$, $P R h ; J-N$, Ins), showed labeling patterns that were consistent with those of the neocortex, inasmuch as there were high levels of TASK-3, TRAAK, and TWIK-1 and very low levels of TREK-2. There were particularly high levels of labeling in the outermost cell body layers; this pattern was seen for TASK-3, TRAAK, and TWIK-1 in the retrosplenial cortex (Fig. $1 E-J$ ), for TWIK-1 in the cingulate cortex (Fig. $1 K-N$ ), and for TASK-3 in the insular cortex (Fig. 1J-N). TREK-1 was expressed at low levels in the perirhinal cortex (Fig. $1 F-I$ ) and was virtually absent from the insular cortex (Fig. $1 J-N)$; this once again was consistent with expression of TREK-1 in layer IV granule neurons of the neocortex, because the perirhinal and insular cortices lack a well developed granule cell layer.

Neurons in the piriform cortex (Pir) were remarkable for the fact that they displayed moderate-to-high labeling of all six transcripts (Fig. $1 H-N$ ). For TREK-1, this labeling was somewhat higher in more rostral sections. Neurons in the claustrum (Fig. $1 \mathrm{~K}-\mathrm{N}, \mathrm{Cl}$ ) and the dorsal endopiriform nucleus (Fig. $1 \mathrm{I}-\mathrm{N}, \mathrm{DEn}$ ) expressed high levels of TASK-3.

\section{Olfactory system}

In the main olfactory bulb $(M O B)$, there once again was substantial overlap of expression (Fig. 1O). Labeling was highest for TASK-1 and TASK-3, and these were the only transcripts found at appreciable levels in the glomerular layer. mRNA for all six genes was detected in olfactory granule cells, with TASK-1 and TASK-3 at moderate-to-high levels but with TRAAK at only low levels. Expression in the anterior olfactory nucleus $(A O)$ was notable for TASK-1, TASK-3, TRAAK, and TWIK-1. In addition, the external segment of this nucleus (data not shown) expressed high levels of TREK-1.

\section{Expression in the dorsal root ganglia}

As noted, all seven genes were expressed in dorsal root ganglia (Fig. 7). TASK-1, TASK-2, and TRAAK each were expressed by a large proportion of the cells; expression of TREK-1, TREK-2, TASK-3, and TWIK-1 was much more restricted, although for each, there were cells with high levels of expression. Expression of TWIK-1 appeared for the most part to be limited to the very large sensory neurons (Fig. $7 H$ ), a pattern also seen in sensory neurons of the mesencephalic trigeminal nucleus (Fig. 3).

\section{KCNK gene expression in the mouse}

In addition to the brain sections taken from rat, a few horizontal and sagittal sections were taken from C57BL/6J mice. Also, mouse brainstem and spinal cord slices were taken for analysis of TASK-1, TASK-3, TREK-1, and TRAAK (data not shown). Although a full distribution could not be obtained with these few sections, it was clear that there were no gross differences in the expression patterns, and all of the regions of high expression were identical between the two species. However, a few subtle differences were apparent. For TASK-3, there may have been somewhat less labeling in the thalamus and cerebellum (relative to other brain regions) in the mouse when compared with the rat. Also, the dorsal-to-ventral gradient of TASK-3 expression that was clearly evident in the rat hippocampus (see above) was less apparent in the mouse when comparing expression in sagittal sections (which contained dorsal hippocampus) with horizontal sections (which contained ventral hippocampus). This was also the case for TWIK-1 expression in CA1 pyramidal neurons. In addition, there was moderate TWIK-1 expression in the striatum of the mouse; labeling in this region was low in the rat. There were a couple of slight differences in expression of TRAAK; in the cortex, it appeared that labeling in mouse sections was somewhat higher in the outermost layers compared with the more even laminar distribution of the rat. In the hippocampus, the TRAAK signal was a bit higher in CA3 pyramidal neurons compared with those of CA1; this once again was in contrast to the more even distribution in the rat. No differences were detectable in the distributions of TASK-1, TASK-2, or TREK-2. The patterns of TREK-1 expression were the same for the two species, except that regions of high TREK-1 expression in the rat (e.g., striatum, layer IV of the neocortex, and CA2 pyramidal neurons) were even 
more strongly labeled relative to other areas in sections from the mouse.

\section{DISCUSSION}

In this study, we describe the CNS distribution of mRNA encoding seven two-pore-domain potassium channel family members. We found widespread and differential distribution of all transcripts except TASK-2, which was expressed at high levels in the dorsal root ganglia but was present only at uniformly low levels in the CNS. The expression of these $\mathrm{KCNK}$ genes was to a large extent complementary; nevertheless, there was substantial overlap of these transcripts in multiple brain areas and likely in individual neurons. Thus, not only do the data suggest that these channels contribute to neuronal diversity, but also that multiple two-pore-domain $\mathrm{K}^{+}$channels may provide contributions to the physiological properties and modulatory potential of individual neurons. In addition, the likely coexpression of different subunits suggests the possibility of coassociation (i.e., the formation of heterodimeric channels), particularly by genes that share higher degrees of sequence homology. However, to date there are no reports of heterodimerization among two-pore-domain $\mathrm{K}^{+}$channel family members.

Currently, 14 two-pore-domain $\mathrm{K}^{+}$channel genes have been identified by molecular cloning, although three (KCNK6/7, THIK-2, and TASK-5) have not generated functional currents in heterologous expression systems (Salinas et al., 1999; Rajan et al., 2000a; Girard et al., 2001; Kim and Gnatenco, 2001). In addition, three (TALK-1, TALK-2/TASK-4, and TASK-5) were cloned very recently from human tissues; they do not appear to be expressed to an appreciable extent in the human brain, and corresponding rodent orthologs have not been identified (Decher et al., 2001; Girard et al., 2001; Kim and Gnatenco, 2001). Of the two remaining genes that we did not examine, TW IK-2 appears to be absent from the rodent brain (Patel et al., 2000). Thus, in the present report, we describe seven of eight functional two-poredomain potassium channel members with known CNS expression. The exception is the recently cloned THIK-1, which along with the presumed channel THIK-2, is expressed in multiple brain regions in the rat (Rajan et al., 2000a; see below).

\section{Comparison with previous reports}

There have been previous brief descriptions of mRNA expression of these channels in the rodent CNS; our more detailed results are to a large extent in agreement with these reports. As with the current work, two reports on TASK-1 show a predominance of mRNA expression in the cerebellum, in the granule cell layer (Duprat et al., 1997; Brickley et al., 2001). For TASK-2, low levels were seen in the CNS by reverse transcription (RT)-PCR, although in contrast to our results, no expression was seen in dorsal root ganglia (Gray et al., 2000) (but see Medhurst et al., 2001). Also in concordance with our results are two recent abstracts reporting widespread CNS distribution of TASK-3 by in situ hybridization, with prominence in many of the same nuclei described here (Rajan et al., 2000b; Vega-Saenz de Miera et al., 2000). Our finding that TREK-2 was primarily restricted to the cerebellum is consistent with previous Northern blot analysis (Bang et al., 2000). Similarly, our findings of high levels of TRAAK in the cerebral cortex and TWIK-1 in the cerebellum and cortex are in concert with previous studies (Lesage et al., 1997; Fink et al., 1998). Also note that a comparison of the current results with studies using Northern blotting and/or RTPCR of tissues dissected from various human brain regions
(Chapman et al., 2000; Lesage et al., 2000; Medhurst et al., 2001) suggests that there is a substantial degree of conservation of expression between humans and rodents. This was particularly evident when considering regions of maximal expression (TWIK-1, cerebellum; TREK-1, caudate putamen; TASK-1, cerebellum; TASK-2, dorsal root ganglia; and TRAAK, cortex).

There were some discrepancies between the current results and previous work, most notably with regard to TREK-1, which we found to be highest in the striatum, CA 2 of the hippocampus, and layer IV of the neocortex. This is in contrast to a previous report in which levels in the striatum were unremarkable, and there was uniform labeling of hippocampal pyramidal neurons and in the various cortical laminas (Fink et al., 1996). The reasons for these and other discrepancies are not clear, although it should be pointed out that the distributions we observed were consistent for rat (using two different strains) and mouse. Furthermore, we used a number of different controls (see Materials and Methods), including the use of multiple oligonucleotide probes (two oligonucleotides each for TREK-1, TRAAK, and TWIK-1 and three oligonucleotides for TASK-1), each of which yielded a distribution pattern that was identical to those of the corresponding cRNA probes.

There are reports of protein localization using antibodies to a few of these channels, including TRAAK (Bearzatto et al., 2000; Lauritzen et al., 2000; Reyes et al., 2000), TREK-1 (Bearzatto et al., 2000; Hervieu et al., 2001; Maingret et al., 2001), and TASK-1 (Kindler et al., 2000; Millar et al., 2000). Complete agreement between protein and mRNA localization is not to be expected for a number of reasons, including the fact that mRNA in most cases is restricted to the cell soma, whereas protein can be transported to sites far from the origin of synthesis (e.g., the axon terminal). In this regard, there is immunohistochemical evidence that both TRAAK and TREK-1 are targeted in part to axon terminals (Bearzatto et al., 2000) and are present at synaptic and nonsynaptic sites in cerebellar cultures (Lauritzen et al., 2000). Despite this, there are many regions in which TRAAK and TREK-1 immunoreactivity correlate with the mRNA expression that we described here. For TRAAK, cell bodies and neuropil were labeled in multiple areas of the mouse brain (Reyes et al., 2000), including the cerebral cortex, a region where we found high levels of mRNA. There were interesting correlations between TREK-1 mRNA expression and immunoreactivity in a number of areas. For example, intense immunoreactivity was seen in the pyramidal layer in CA2 of the hippocampus (Hervieu et al., 2001), suggesting that the high levels of mRNA expression that we found in these neurons results in protein expression at the cell body and proximal dendrites. Similarly, immunoreactivity was seen on the cell bodies of GABAergic neurons in the striatum (Hervieu et al., 2001), another region with high levels of TREK-1 mRNA.

An antibody to TASK-1 also is available; however, in our own work (E. M. Talley and D. A. Bayliss, unpublished observations) and in a published report (Kindler et al., 2000) (see also Millar et al., 2000), it strongly labeled astrocytes, including those located in fiber tracts such as the external capsule and corpus callosum. As noted (see Results), we found no evidence for TASK-1 mRNA in any fiber tracts. Furthermore, for most neuronal populations, this antibody only gave a faint signal, and there was no labeling of motoneurons, where we found high levels of TASK-1 mRNA and where there is strong evidence for functional expression of TASK-1 at the cell soma (Sirois et al., 2000; Talley et al., 2000). The reasons for these discrepancies are not clear. 


\section{Regions of low expression}

There were a number of regions in which there was little expression of any of these transcripts (e.g., the substantia nigra and inferior olivary nucleus). In these and other regions, it is likely that other channels provide requisite $\mathrm{K}^{+}$conductances for maintenance of baseline membrane properties. In this regard, it is important to consider the other two-pore-domain family channels that were not a part of this study (see above). Of these, two are known to be expressed in the CNS at appreciable levels, THIK-1 and the presumed channel THIK-2. However, neither of these channels appears to be enriched in regions identified here as showing low expression for other two-pore-domain $\mathrm{K}^{+}$channel transcripts (Rajan et al., 2000a). Note also that baseline potassium conductances can receive contributions from members of the $\mathrm{K}_{\mathrm{IR}}$ and $\mathrm{K}_{\mathrm{V}}$ families (for example, see Williams et al., 1988; Karschin et al., 1996). Indeed, a member of the $K_{V}$ family (KCNQ1) forms a constitutively active leak channel when coexpressed with certain regulatory subunits (Schroeder et al., 2000; Tinel et al., 2000).

\section{Functional correlates in the CNS}

Our results support the hypothesis that, as a result of selective expression, heterogeneity among two-pore-domain $\mathrm{K}^{+}$channels contributes to variation in the physiological properties and modulation of different populations of neurons. Consistent with our results, TASK-like currents have been identified in motoneurons and locus ceruleus neurons, as well as cerebellar granule neurons, by virtue of their sensitivity to $\mathrm{pH}$, anesthetics, and (in the case of granule neurons) the endocannabinoid anandamide (Millar et al., 2000; Sirois et al., 2000; Talley et al., 2000; Maingret et al., 2001). In addition, arachadonic acid, which activates TREK-1, TREK-2, and TRAAK, potentiates baseline $\mathrm{K}^{+}$currents in cerebellar granule cells (Lauritzen et al., 2000) and hippocampal pyramidal neurons (Colbert and Pan, 1999). In neurons dissociated from fetal mesencephalon and hypothalamus (Kim et al., 1995), three types of fatty acid- and stretch-activated $\mathrm{K}^{+}$channels were identified based on single-channel properties, two of which appear to correspond to TREK-1 and TREK-2, respectively (Fink et al., 1996; Maingret et al., 1999; Bang et al., 2000; Lesage et al., 2000). Also consistent with expression of TREK-1, which is strongly sensitive to temperature changes below $37^{\circ} \mathrm{C}$ (Maingret et al., 2000), background $\mathrm{K}^{+}$currents have been implicated in the actions of cold-responsive sensory neurons of the dorsal root ganglia (Reid and Flonta, 2001). Finally, a host of neuronal types are subject to effects of neurotransmitter inhibition of background $\mathrm{K}^{+}$currents (McCormick, 1992; Rekling et al., 2000), although correlation with specific channel types has only been made in the case of motoneurons and cerebellar granule neurons (Millar et al., 2000; Talley et al., 2000).

In addition to endogenous substances, a number of two-poredomain $\mathrm{K}^{+}$channels are responsive to low concentrations of volatile anesthetics such as halothane and sevofluorane (Patel et al., 1999; Gray et al., 2000; Lesage et al., 2000; Rajan et al., 2000a; Sirois et al., 2000). It has been proposed that clinical actions of these compounds may result in part from activation of TASK channels in motoneurons and in locus ceruleus neurons (Sirois et al., 2000), where inhibition of firing would contribute to immobilizing and hypnotic effects, respectively. Also, two-pore-domain channels have been proposed as substrates for neuroprotective agents, including some cellular lipids (Fink et al., 1998; Duprat et al., 2000; Lauritzen et al., 2000; Meadows et al., 2001). Thus, the value of these channels as targets for therapeutically important compounds already is evident, and the development of more selective agents may be of more than experimental benefit.

\section{REFERENCES}

Bang H, Kim Y, Kim D (2000) TREK-2, a new member of the mechanosensitive tandem-pore $\mathrm{K}^{+}$channel family. $\mathrm{J}$ Biol Chem 275:17412-17419.

Bearzatto B, Lesage F, Reyes R, Lazdunski M, Laduron PM (2000) Axonal transport of TREK and TRAAK potassium channels in rat sciatic nerves. NeuroReport 11:927-930.

Brickley SG, Revilla V, Cull-Candy SG, Wisden W, Farrant M (2001) Adaptive regulation of neuronal excitability by a voltage-independent potassium conductance. Nature 409:88-92.

Chapman CG, Meadows HJ, Godden RJ, Campbell DA, Duckworth M, Kelsell RE, Murdock PR, Randall AD, Rennie GI, Gloger IS (2000) Cloning, localisation and functional expression of a novel human, cerebellum specific, two pore domain potassium channel. Brain Res Mol Brain Res 82:74-83.

Colbert CM, Pan E (1999) Arachidonic acid reciprocally alters the availability of transient and sustained dendritic $\mathrm{K}^{+}$channels in hippocampal CA1 pyramidal neurons. J Neurosci 19:8163-8171.

Decher N, Maier M, Dittrich W, Gassenhuber J, Bruggemann A, Busch AE, Steinmeyer K (2001) Characterization of TASK-4, a novel member of the pH-sensitive, two-pore domain potassium channel family. FEBS Lett 492:84-89.

Duprat F, Lesage F, Fink M, Reyes R, Heurteaux C, Lazdunski M (1997) TASK, a human background $\mathrm{K}^{+}$channel to sense external $\mathrm{pH}$ variations near physiological pH. EMBO J 16:5464-5471.

Duprat F, Lesage F, Patel AJ, Fink M, Romey G, Lazdunski M (2000) The neuroprotective agent riluzole activates the two $\mathrm{P}$ domain $\mathrm{K}^{+}$ channels TREK-1 and TRAAK. Mol Pharmacol 57:906-912.

Fink M, Duprat F, Lesage F, Reyes R, Romey G, Heurteaux C, Lazdunski M (1996) Cloning, functional expression and brain localization of a novel unconventional outward rectifier $\mathrm{K}^{+}$channel. EMBO J 15:6854-6862.

Fink M, Lesage F, Duprat F, Heurteaux C, Reyes R, Fosset M, Lazdunski M (1998) A neuronal two $\mathrm{P}$ domain $\mathrm{K}^{+}$channel stimulated by arachidonic acid and polyunsaturated fatty acids. EMBO J 17:3297-3308.

Girard C, Duprat F, Terrenoire C, Tinel N, Fosset M, Romey G, Lazdunski M, Lesage F (2001) Genomic and functional characteristics of novel human pancreatic $2 \mathrm{P}$ domain $\mathrm{K}^{+}$channels. Biochem Biophys Res Commun 282:249-256.

Goldstein SA, Bockenhauer D, O'Kelly I, Zilberberg N (2001) Potassium leak channels and the KCNK family of two-P-domain subunits. Nat Rev Neurosci 2:175-184.

Gray AT, Zhao BB, Kindler CH, Winegar BD, Mazurek MJ, Xu J, Chavez RA, Forsayeth JR, Yost CS (2000) Volatile anesthetics activate the human tandem pore domain baseline $\mathrm{K}^{+}$channel KCNK5. Anesthesiology 92:1722-1730.

Hervieu GJ, Cluderay JE, Gray CW, Green PJ, Ranson JL, Randall AD, Meadows HJ (2001) Distribution and expression of TREK-1, a twopore-domain potassium channel, in the adult rat CNS. Neuroscience 103:899-919.

Karschin C, Dissmann E, Stuhmer W, Karschin A (1996) IRK(1-3) and GIRK(1-4) inwardly rectifying $\mathrm{K}^{+}$channel mRNAs are differentially expressed in the adult rat brain. J Neurosci 16:3559-3570.

Kim D, Gnatenco C (2001) TASK-5, a new member of the tandem-pore $\mathrm{K}^{+}$channel family. Biochem Biophys Res Commun 284:923-930.

Kim D, Sladek CD, Aguado-Velasco C, Mathiasen JR (1995) Arachidonic acid activation of a new family of $\mathrm{K}^{+}$channels in cultured rat neuronal cells. J Physiol (Lond) 484:643-660.

Kim Y, Bang H, Kim D (2000) TASK-3, a new member of the tandem pore $\mathrm{K}^{+}$channel family. J Biol Chem 275:9340-9347.

Kindler CH, Pietruck C, Yost CS, Sampson ER, Gray AT (2000) Localization of the tandem pore domain $\mathrm{K}^{+}$channel TASK-1 in the rat central nervous system. Brain Res Mol Brain Res 80:99-108.

Lauritzen I, Blondeau N, Heurteaux C, Widmann C, Romey G, Lazdunski M (2000) Polyunsaturated fatty acids are potent neuroprotectors. EMBO J 19:1784-1793.

Leonoudakis D, Gray AT, Winegar BD, Kindler CH, Harada M, Taylor DM, Chavez RA, Forsayeth JR, Yost CS (1998) An open rectifier potassium channel with two pore domains in tandem cloned from rat cerebellum. J Neurosci 18:868-877.

Lesage F, Lazdunski M (2000) Molecular and functional properties of two-pore-domain potassium channels. Am J Physiol 279:F793-F801.

Lesage F, Guillemare E, Fink M, Duprat F, Lazdunski M, Romey G, Barhanin J (1996) TW IK-1, a ubiquitous human weakly inward rectifying $\mathrm{K}^{+}$channel with a novel structure. EMBO J 15:1004-1011.

Lesage F, Lauritzen I, Duprat F, Reyes R, Fink M, Heurteaux C, Lazdunski M (1997) The structure, function and distribution of the mouse TWIK-1 K ${ }^{+}$channel. FEBS Lett 402:28-32.

Lesage F, Terrenoire C, Romey G, Lazdunski M (2000) Human TREK2, a 2P domain mechano-sensitive $\mathrm{K}^{+}$channel with multiple 
regulations by polyunsaturated fatty acids, lysophospholipids, and Gs, Gi, and Gq protein-coupled receptors. J Biol Chem 275:28398-28405.

Maingret F, Patel AJ, Lesage F, Lazdunski M, Honore E (1999) Mechano- or acid stimulation, two interactive modes of activation of the TREK-1 potassium channel. J Biol Chem 274:26691-26696.

Maingret F, Lauritzen I, Patel AJ, Heurteaux C, Reyes R, Lesage F, Lazdunski M, Honore E (2000) TREK-1 is a heat-activated background $\mathrm{K}^{+}$channel. EMBO J 19:2483-2491.

Maingret F, Patel AJ, Lazdunski M. Honore E (2001) The endocannabinoid anandamide is a direct and selective blocker of the background $\mathrm{K}^{+}$channel TASK-1. EMBO J 20:47-54.

McCormick DA (1992) Neurotransmitter actions in the thalamus and cerebral cortex and their role in neuromodulation of thalamocortical activity. Prog Neurobiol 39:337-388.

Meadows HJ, Chapman CG, Duckworth DM, Kelsell RE, Murdock PR, Nasir S, Rennie G, Randall AD (2001) The neuroprotective agent sipatrigine (BW619C89) potently inhibits the human tandem poredomain $\mathrm{K}^{+}$channels TREK-1 and TRAAK. Brain Res 892:94-101.

Medhurst AD, Rennie G, Chapman CG, Meadows H, Duckworth MD, Kelsell RE, Gloger II, Pangalos MN (2001) Distribution analysis of human two pore domain potassium channels in tissues of the central nervous system and periphery. Brain Res Mol Brain Res 86:101-114.

Millar JA, Barratt L, Southan AP, Page KM, Fyffe RE, Robertson B, Mathie A (2000) A functional role for the two-pore domain potassium channel TASK-1 in cerebellar granule neurons. Proc Natl Acad Sci USA 97:3614-3618.

Patel AJ, Honore E (2001) Properties and modulation of mammalian 2P domain $\mathrm{K}^{+}$channels. Trends Neurosci 24:339-346.

Patel AJ, Honore E, Lesage F, Fink M, Romey G, Lazdunski M (1999) Inhalational anesthetics activate two-pore-domain background $\mathrm{K}^{+}$ channels. Nat Neurosci 2:422-426.

Patel AJ, Maingret F, Magnone V, Fosset M, Lazdunski M, Honore E (2000) TWIK-2, an inactivating $2 \mathrm{P}$ domain $\mathrm{K}^{+}$channel. J Biol Chem 275:28722-28730.

Paxinos G (1995) The rat nervous system. San Diego: Academic.

Paxinos G, Watson C (1997) The rat brain in stereotaxic coordinates. San Diego: Academic.
Rajan S, Wischmeyer E, Karschin C, Preisig-Muller R, Grzeschik KH Daut J, Karschin A, Derst C (2000a) THIK-1 and THIK-2, a novel subfamily of tandem pore domain $\mathrm{K}^{+}$channels. J Biol Chem 275:16650-16657.

Rajan S, Wischmeyer E, Karschin C, Liu GX, Preisig-Muller R, Daut J, Karschin A, Derst C (2000b) TASK-3, a novel brain-specific tandem pore-domain potassium channel. Pflügers Arch 439:R382.

Reid G, Flonta M (2001) Cold transduction by inhibition of a background potassium conductance in rat primary sensory neurones. Neurosci Lett 297:171-174.

Rekling JC, Funk GD, Bayliss DA, Dong XW, Feldman JL (2000) Synaptic control of motoneuronal excitability. Physiol Rev 80:399-410.

Reyes R, Lauritzen I, Lesage F, Ettaiche M, Fosset M, Lazdunski M (2000) Immunolocalization of the arachidonic acid and mechanosensitive baseline TRAAK potassium channel in the nervous system. Neuroscience 95:893-901.

Salinas M, Reyes R, Lesage F, Fosset M, Heurteaux C, Romey G, Lazdunski M (1999) Cloning of a new mouse two-P domain channel subunit and a human homologue with a unique pore structure. J Biol Chem 274:11751-11760.

Schroeder BC, Waldegger S, Fehr S, Bleich M, Warth R, Greger R, Jentsch TJ (2000) A constitutively open potassium channel formed by KCNQ1 and KCNE3. Nature 403:196-199.

Sirois JE, Lei Q, Talley EM, Lynch C, Bayliss DA (2000) The TASK-1 two-pore domain $\mathrm{K}^{+}$channel is a molecular substrate for neuronal effects of inhalation anesthetics. J Neurosci 20:6347-6354.

Talley EM, Lei Q, Sirois JE, Bayliss DA (2000) TASK-1, a two-pore domain $\mathrm{K}^{+}$channel, is modulated by multiple neurotransmitters in motoneurons. Neuron 25:399-410.

Tinel N, Diochot S, Borsotto M, Lazdunski M, Barhanin J (2000) KCNE2 confers background current characteristics to the cardiac KCNQ1 potassium channel. EMBO J 19:6326-6330.

Vega-Saenz de Miera E, Ozaita A, Zadina M, Rudy B (2000) Expression of two pore $\mathrm{K}^{+}$channels in the CNS. Soc Neurosci Abstr 26:685.7

Williams JT, Colmers WF, Pan ZZ (1988) Voltage- and ligand-activated inwardly rectifying currents in dorsal raphe neurons in vitro. J Neurosci 8:3499-3506. 